\title{
Gemini spectroscopy of the outer disk star cluster BH176^
}

\author{
M. E. Sharina ${ }^{1, \star \star}$, C. J. Donzelli ${ }^{2}$, E. Davoust ${ }^{3}$, V. V. Shimansky ${ }^{4}$, and C. Charbonnel ${ }^{5}$ \\ ${ }^{1}$ Special Astrophysical Observatory, Russian Academy of Sciences, N. Arkhyz, KChR, 369167, Russia \\ e-mail: sme@sao.ru \\ 2 Instituto de Astronomía Teórica y Experimental IATE, CONICET - Observatorio Astronómico, Universidad Nacional de Córdoba, \\ Laprida 854, X5000BGR Córdoba, Argentina \\ e-mail: charly@oac.uncor.edu \\ 3 IRAP, Université de Toulouse, CNRS, 14 avenue E. Belin, 31400 Toulouse, France \\ e-mail: edavoust@irap.omp.eu \\ ${ }^{4}$ Kazan Federal University, Kremlevskaya 18, 420008 Kazan, Russia \\ e-mail: Slava. Shimansky@kpfu.ru \\ 5 Geneva Observatory, University of Geneva, 51 Ch. des Maillettes, 1290 Versoix, Switzerland \\ e-mail: corinne.charbonnel@unige.ch
}

Received 12 February 2014 / Accepted 25 July 2014

\section{ABSTRACT}

\begin{abstract}
Context. BH176 is an old metal-rich star cluster. It is spatially and kinematically consistent with belonging to the Monoceros Ring. It is larger in size and more distant from the Galactic plane than typical open clusters, and it does not belong to the Galactic bulge. Aims. Our aim is to determine the origin of this unique object by accurately determining its distance, metallicity, and age. The best way to reach this goal is to combine spectroscopic and photometric methods.

Methods. We present medium-resolution observations of red clump and red giant branch stars in BH176 obtained with the Gemini South Multi-Object Spectrograph. We derive radial velocities, metallicities, effective temperatures, and surface gravities of the observed stars and use these parameters to distinguish member stars from field objects.

Results. We determine the following parameters for BH176: $V_{\mathrm{h}}=0 \pm 15 \mathrm{~km} \mathrm{~s}^{-1},[\mathrm{Fe} / \mathrm{H}]=-0.1 \pm 0.1$, age $7 \pm 0.5 \mathrm{Gyr}$, $E(V-I)=0.79 \pm 0.03$, distance $15.2 \pm 0.2 \mathrm{kpc}, \alpha$-element abundance $[\alpha / \mathrm{Fe}] \sim 0.25$ dex (the mean of $[\mathrm{Mg} / \mathrm{Fe}]$, and $[\mathrm{Ca} / \mathrm{Fe}]$ ).

Conclusions. BH176 is a member of old Galactic open clusters that presumably belong to the thick disk. It may have originated as a massive star cluster after the encounter of the forming thin disk with a high-velocity gas cloud or as a satellite dwarf galaxy.
\end{abstract}

Key words. globular clusters: general - globular clusters: individual: BH176 - open clusters and associations: general Galaxy: structure - Galaxy: formation

\section{Introduction}

Star clusters (SCs) are important tracers of the evolutionary history of our Galaxy. The knowledge of their chemical, structural, and dynamical properties is indispensable for understanding how the Galaxy formed and how much dwarf satellites contributed to its formation. The age, distance, and metal content of SCs can be determined more easily than for single stars. SCs are prime targets for improving our understanding of stellar evolution using deep colour-magnitude diagrams (CMD) and high-resolution spectroscopy. The old globular clusters (GCs), which populate galactic haloes, were enriched in $\alpha$-elements during their formation due to explosions of SNeII (Kruijssen \& Diederik, and references therein). The high-metallicity GCs

\footnotetext{
$\star$ Appendix A is available in electronic form at http: //www . aanda.org

$\star \star$ Based on observations obtained at the Gemini Observatory, which is operated by the Association of Universities for Research in Astronomy, Inc., under a cooperative agreement with the NSF on behalf of the Gemini partnership: the National Science Foundation (United States), the Science and Technology Facilities Council (United Kingdom), the National Research Council (Canada), CONICYT (Chile), the Australian Research Council (Australia), Ministério da Ciência, Tecnologia e Inovação (Brazil), and the Ministerio de Ciencia, Tecnología e Innovación Productiva (Argentina).
}

$([\mathrm{Fe} / \mathrm{H}]>-0.4$ dex $)$ belong to the bulge or the thick disk, but their origin is still an open subject.

Open clusters $(\mathrm{OCs})$ reside in the thin disk. They are generally less massive and more metal-rich than GCs and lose stars from tidal interactions with the interstellar medium. Because of the enormous extinction and density of matter near the Galactic plane and because of their loose structure, OCs have only been studied within a radius of $R_{\mathrm{gc}} \sim 8 \mathrm{kpc}$ from the Sun (Kharchenko et al. 2013). Young embedded SCs presently undergoing star formation have been discovered in dense molecular clouds. Understanding the mechanisms triggering the formation of massive SCs is one of the primary goals of contemporary astrophysics.

The nature of BH176 $\left(\alpha=15^{\mathrm{h}} 39^{\mathrm{m}} 07.3, \delta=-50^{\circ} 03^{\prime} 02^{\prime \prime}\right.$, J2000.0), which was discovered and classified as an OC by van den Bergh \& Hagen (1975), is still a matter of debate. The stellar populations of the SC were studied by photometric (Ortolani et al. 1995; Phelps \& Schick 2003) and by photometric and spectroscopic methods (Frinchaboy et al. 2006). The latter provided the first reliable radial velocity $\left(V_{\mathrm{h}}=11.2 \pm 5.3 \mathrm{~km} \mathrm{~s}^{-1}\right)$, distance from the Sun $(15.8 \mathrm{kpc})$, photometric metallicity $([\mathrm{Fe} / \mathrm{H}]$ $0.0 \mathrm{dex}$ ), and age (7 Gyr) for BH176. Their conclusion was that the SC is a possible member of the Monoceros stream according to its location and kinematics; however, the high metallicity suggests that this connection is unlikely. The metallicity distribution of the Monoceros stream was measured by 
Meisner et al. (2012) from the spectroscopy of 594 stars obtained with the low-resolution MMT Hectospec. They found the stream to be chemically distinct from both the thick disk and halo, with $[\mathrm{Fe} / \mathrm{H}]=-1$ dex and a small intrinsic dispersion of $0.10 \div 0.22$ dex. BH176 does not fit the age-metallicity relation of the Sgr dwarf spheroidal galaxy (Layden \& Sarajedini 2000). Harris (1996) classified this SC as a GC with a distance of $15.6 \mathrm{kpc}$ from the Sun, extinction $E(B-V)=0.77 \mathrm{mag}$, visual level of the horizontal branch $V_{\mathrm{HB}}=19.0 \mathrm{mag}$, and total visual absolute magnitude $M_{V}=-4.35 \mathrm{mag}$.

Davoust et al. (2011, hereafter D11) used another approach to redetermine the age and metallicity of this unique object and to accurately derive its distance and Galactic extinction using the Two Micron All Sky Survey (2MASS) Point Source catalogue and archival VLT images. Red giant branch (RGB) stars belonging to BH176 were detected independently in the visual and infrared wavelength ranges in the region of the CMDs defined by the following equations: $K_{\mathrm{s} 0}<-9.09\left(J-K_{\mathrm{s}}\right)_{0}+20.0$, $I<-6.9(V-I)+29$, and $V-I>1.8$ (where $V$ and $I$ magnitudes are not corrected for Galactic extinction). The spatial distribution of stars selected in this way allowed the authors to derive a maximum extent of $3^{\prime}$ for the cluster.

Data from the 2MASS Point Source Catalog with a good photometric quality allowed them to reach a photometric limit of $\sim 5$ mag below the tip of the RGB (TRGB) in the $K_{\mathrm{s}}$ band. The strong Galactic extinction was not an obstacle in the infrared; however, owing to the poor photometric depth and the large number of foreground stars, it was difficult to derive the distance accurately. There are only three red giant stars within the boundaries of BH176 3 mag from the TRGB $\left(K_{\mathrm{s}}=[12 \div 9]\right)$. The derived extinction in the $K_{\mathrm{s}}$ band was $A_{K_{\mathrm{s}}}=0.212 \pm 0.03 \mathrm{mag}$. Artificial star tests were made in the course of the stellar photometry on the VLT images to evaluate how the crowding influences the photometric depth and accuracy. The deep visual CMD reached $\sim 1$ mag below the main sequence turn-off (MSTO) of the SC. However, owing to strong contamination problems the ridge line outlining the upper MS, SGB, and RGB of the SC was defined only statistically. Since the MSTO was close to the photometric limit and strongly contaminated by foreground stars and because of the scarcity of stars on the TRGB, only RC stars provided a good distance indicator representing a strong peak in the colour-magnitude density distribution.

Using theoretical predictions for the dependence of the $I$-band magnitude and $(V-I)_{0}$ colour of the RC on the age and metallicity (Girardi \& Salaris 2001), the following parameters were obtained for BH176: $[\mathrm{Fe} / \mathrm{H}]=-0.17_{-0.11}^{+0.08} \mathrm{dex}^{1}$, $M_{V}(\mathrm{RC})=0.88 \pm 0.07, M_{I}(\mathrm{RC})=-0.17 \pm 0.07$, and $(m-M)_{0}=$ $15.9 \pm 0.07$. Combining these data with the ones derived using different methods (isochrone fitting, RGB bump luminosity) led to the following results: Dist $=15.1 \pm 0.5 \mathrm{kpc}, E(V-I)=0.796$, $[\mathrm{Fe} / \mathrm{H}]=-0.1 \pm 0.1$ dex, age $=7$ Gyr. The authors suggested that BH176 is an open, or transitional cluster. We simply use the term "star cluster" throughout this paper. Indeed, BH176 has properties that are distinct from both OCs and GCs, but the term "transitional cluster" has not been clearly defined.

In this study we present and analyse data from the Gemini South Multi-Object Spectrograph for RGB and RC stars in BH176, selected using the above photometric study (D11). The pre-imaging run and spectroscopic observations are described in Sect. 2. The reduction method and spectroscopic results are

\footnotetext{
$1[\mathrm{Fe} / \mathrm{H}]=\log (\mathrm{Fe} / \mathrm{H})_{*}-\log (\mathrm{Fe} / \mathrm{H})_{\odot}$, where $\log (\mathrm{Fe})=$ $\log (n(\mathrm{Fe}) / n(\mathrm{H}))+12$ and $n(\mathrm{Fe})$ and $n(H)$ are the numerical densities $\left(\mathrm{cm}^{-3}\right)$ of iron and hydrogen atoms, respectively.
}

Table 1. Journal of pre-imaging and spectroscopic observations.

\begin{tabular}{lccccr}
\hline \hline Object & Date & Filter/grism & $\begin{array}{c}T_{\exp } \\
(\mathrm{s})\end{array}$ & $\begin{array}{c}\text { Seeing } \\
(\operatorname{arcsec})\end{array}$ & Air mass \\
\hline pre image & $2011-02-01$ & G0325 & $1 \times 60$ & 0.95 & 1.39 \\
mask 1 & $2011-03-29$ & B600-G5323 & $3 \times 1200$ & 0.94 & 1.09 \\
mask 2 & $2011-03-30$ & B600-G5323 & $2 \times 1200$ & 0.98 & 1.14 \\
mask 2 & 2011-04-02 & B600-G5323 & $1 \times 1200$ & 0.98 & 1.16 \\
\hline
\end{tabular}

summarised in Sect. 3. A short Sect. 4 is devoted to the propermotion issues. In Sect. 5 isochrones are fit to the VLT photometric data to improve the extinction, distance, and age estimates. The results are discussed in Sect. 6 and summarised in Sect. 7.

\section{Observations and data reduction}

The spectroscopic observations were carried out with the Gemini South telescope using the Gemini Multi-Object Spectrograph (GMOS) as part of Gemini programme GS-2011A-Q-22 (PI: Donzelli). The targets selected within the boundaries of BH176 generally follow the ridge line outlined by the VLT stellar photometry of D11. These are bright stars falling on the possible positions of the slits with magnitudes and colours roughly corresponding to the RC and RGB of the SC. To use all the slits, some field stars with $V-I<1.8$ were also observed.

\subsection{Images}

To set the slits accurately (see next section) a $60 \mathrm{~s}$ exposure in the SDSS $g^{\prime}$ filter was obtained (see Table 1 for details). The field covers a region of $5 \times 5 \mathrm{arcmin}^{2}$, with a pixel scale of 0.145 arcsec, which is perfectly suitable for our targets. The image was provided by the Gemini staff, and it was automatically reduced by the Gemini pipeline. Two GMOS multi-slit masks were designed with the GMOS Mask Making software using this preliminary image. The image was also used to transform the VLT coordinates of the target stars into the original astrometric system of the Gemimi South telescope.

\subsection{Spectra}

Using the B600-G5323 grating blazed at $5000 \AA$, a slit width of 1 arcsec and a minimum slit length of 4 arcsec, we were able to allocate 41 and 39 slits in masks 1 and 2, respectively. In each case, a total of $3 \times 1200 \mathrm{~s}$ exposures were obtained at three different central wavelengths $(4970,5020$, and $5070 \AA)$ in order to correct for the gaps between CCDs. A binning of $2 \times 2$ was used, yielding a scale of 0.146 arcsec pixel ${ }^{-1}$, a theoretical dispersion of $\sim 0.9 \AA$ per pixel, and a resolution of $F W H M \sim 5 \AA$. The spectroscopic data were acquired in queue mode under very good seeing conditions (see Table 1 for details).

The spectra cover the range 3600-6300 $\AA$, but the exact range depends on the slit position on the mask. Bias frames, dome, and sky flat fields and Copper-argon (CuAr) arcs were taken for calibration. Wavelength calibration left residuals of $0.2 \AA$. Flux calibration was performed using the spectra of the standard star LTT9239, which was obtained with the same instrument configuration as for the science objects. All science and calibration files were retrieved from the Gemini Science Archive hosted by the Canadian Astronomy Data Centre. 
The data were reduced with the Gemini/GMOS package within IRAF following the standard procedure. We normalised the spectroscopic flat fields with GSFLAT, and then mosaicked the three GMOS CCDs into one image with GMOSAIC. We subtracted bias frames and flat-fielded the mosaic object frames using the task GSREDUCE. In the case of the arc frames, we only did the bias subtraction. The procedure GSWAVELENGTH was used to determine the wavelength calibration from the $\mathrm{CuAr}$ spectra. Then the object frames were wavelength-calibrated using the task GSTRANSFORM. Once the object frames were calibrated we did the sky subtraction using GSSKYSUB. The one-dimensional individual spectra were extracted using GSEXTRACT, and the final flux calibration was done with the GSCALIBRATE routine. Prior to the flux calibration, the task GSSTANDARD was used to derive the sensitivity function.

\section{Analysis of the spectroscopic data}

To identify stars belonging to BH176 among our sample, we inspected theoretical and empirical stellar libraries for possible analogues. The main disadvantage of empirical stellar libraries (and stellar population models based on them) is that the abundance patterns are biased towards the solar neighbourhood and low-mass stars. Theoretical spectra are not limited in atmospheric parameters, wavelength range, spectral resolution, or abundance patterns. However, their computation is based on incomplete lists of atomic and molecular lines and inaccurate atomic constants (see e.g. Coelho 2014; Husser et al. 2013, for a thorough discussion).

Preliminary effective temperatures for the stars were estimated using the $\left(V-K_{\mathrm{s}}\right)_{0}$ colour indices and the temperature calibrations by Alonso et al. (1999). These data are listed in Col. 7 of Table A.1. The successive steps of our spectroscopic analysis were the following. First, we compared spectra of the observed stars in and around BH176 with the ELODIE stellar library (Prugniel \& Soubiran 2001; Prugniel et al. 2007). It provides a wide coverage of atmospheric parameters: $T_{\text {eff }}=$ $3100 \mathrm{~K} \div 50000 \mathrm{~K}, \log g=-0.25 \div 4.9$, and $[\mathrm{Fe} / \mathrm{H}]=-3 \div 1 \mathrm{dex}$. Most stars in this library have solar, or near solar, metallicity, close to the one of BH176 (D11). Second, using the effective temperatures, surface gravities, metallicities, and radial velocities derived in the previous step, we developed criteria to separate stars belonging to BH176 from the field ones. Then we co-added spectra of the selected objects to obtain a representative spectrum of high signal-to-noise ratio $(\mathrm{S} / \mathrm{N})$, which was compared with 1) individual stars with similar physical parameters in the MILES stellar library (Sánchez-Blázquez et al. 2006; Cenarro et al. 2007) and 2) synthetic spectra calculated from stellar atmosphere models with various chemical compositions corresponding to the predefined atmospheric parameters.

\subsection{Full spectrum fitting in ULYSS}

To derive the properties of the stars observed in BH176 we used the ULYSS program ${ }^{2}$ (see e.g. Koleva et al. 2009, for a detailed explanation of the software) with the interpolating arrow built on the ELODIE 3.2 stellar library ${ }^{3}$. The ULYSS program performs a non-linear least-squares minimisation of the difference between the model and observed spectra, together with a normalisation of the pseudo-continuum and taking the line-spread

\footnotetext{
2 http://ulyss.univ-lyon $1 . \mathrm{fr}$

3 http://www.obs.u-bordeaux1.fr/m2a/soubiran/elodie_library.html; http://ulyss.univ-lyon 1.fr/models.html
}

function of the spectrograph into account. Multiplicative and additive polynomials were applied to the observed spectrum to bring it in agreement with the model spectrum. Prugniel et al. (2011) and Wu et al. (2011) determined the atmospheric parameters $T_{\text {eff }}, \log (g)$ and $[\mathrm{Fe} / \mathrm{H}]$ for stars in the MILES and Coud-feed Indo-US spectral libraries with this method.

Before the fit we derived line-spread functions for each of the observed slits using twilight spectra obtained at the same positions in the field of view. The reference spectra were degraded to the resolution of the observed spectra.

The derived stellar parameters are listed in Table A.1. The columns contain the following information: (1) slit number (a stands for mask 1, b stands for mask 2); (2) equatorial coordinate (J2000.0); $(3,4)$ visual magnitude in the $I$-band and $(V-I)$ colour from D11, uncorrected for Galactic extinction; $(5,6) K_{\mathrm{s}}$ magnitude and $\left(J-K_{\mathrm{s}}\right)$ colour from the 2MASS Point Source Catalog; (7) $T_{\text {eff }}$, in Kelvin, calculated from $(V-K)_{0}$ according to the empirical calibration of Alonso et al. (1999); (8) distance from the centre of the SC, in arcsec; (9) heliocentric radial velocity with uncertainty, in $\mathrm{km} \mathrm{s}^{-1}$; (10) metallicity $[\mathrm{Fe} / \mathrm{H}]$, in dex; (11) effective temperature, in Kelvin, estimated in ULYSS; $(12)$ surface gravity, in $\mathrm{cm} / \mathrm{s}^{2} ;(13,14)$ proper motion in right ascension $\mu_{\alpha} * \cos (\delta)$ and declination $\mu_{\delta}$ from the PPMXL catalogue (Roeser et al. 2010), in mas $\mathrm{yr}^{-1}$; (15) S/N per resolution element at 5000 in the reduced one-dimensional spectrum; (16) note on the stellar type.

The following information is collected in the last column of Table A.1. The stars belonging to BH176 are marked by an asterisk (RC), or a double asterisk in the case of RGB stars. Three stars were classified as semi-regular variables (SRb; see Sect. 3.3). TiO molecular bands were detected in some RGB stars. The $\mathrm{MgH}$ molecular bands were much stronger in three field stars in comparison to other sample objects. Some objects were difficult to classify due to insufficient $\mathrm{S} / \mathrm{N}$ or to narrow spectral ranges. They are marked by “?”.

\subsection{Identying stars belonging to $\mathrm{BH} 176$}

The distribution of the derived values of $V_{\mathrm{h}}$ and $[\mathrm{Fe} / \mathrm{H}]$ for all the stars observed spectroscopically is shown in Fig. 1 and compared with the density distribution of the corresponding data calculated using the Besançon model of our Galaxy ${ }^{4}$ (Robin et al. 2003, 2004). It also shows the stars classified as belonging to BH176 (marked by asterisks in Table A.1). The following parameters were chosen to generate a catalogue of pseudo-stars in the Johnson-Cousins photometric system with the Besançon model: a distance interval $14.8-16(\mathrm{kpc})$, an area $1 \mathrm{deg}^{2}$ centred at $l=328^{\circ}$ and $b=4^{\circ}$ in Galactic coordinates; magnitudes in the range $23 \geq V \geq 15$ and $21 \geq I \geq 13 ;-200 \geq V_{\mathrm{h}} \geq 100$. The diffuse extinction parameter was set equal to $0.7 \mathrm{mag} \mathrm{kpc}^{-1}$. The default values were adopted for the other parameters (spectral and luminosity types, photometric uncertainties, proper motions). We then selected the stars with $-1.7 \geq[\mathrm{Fe} / \mathrm{H}] \geq 0.7$. The resulting sample contained 9635 stars. Figure 1 shows the distribution of this artificial sample in heliocentric radial velocity and metallicity in the form of numbers of stars per unit bin with a size of $V_{\mathrm{h}}=5 \mathrm{~km} \mathrm{~s}^{-1}$ and $[\mathrm{Fe} / \mathrm{H}]=0.05$ dex. If we select as small an area as possible to fit the position and the size of BH176, the $V_{\mathrm{h}}$ and $[\mathrm{Fe} / \mathrm{H}]$ distribution generated by the Besançon model does not change significantly. We used an area that is large enough to obtain a statistically representative sample.

4 http://model.obs\$-\$besancon.fr/modele_-options.html 

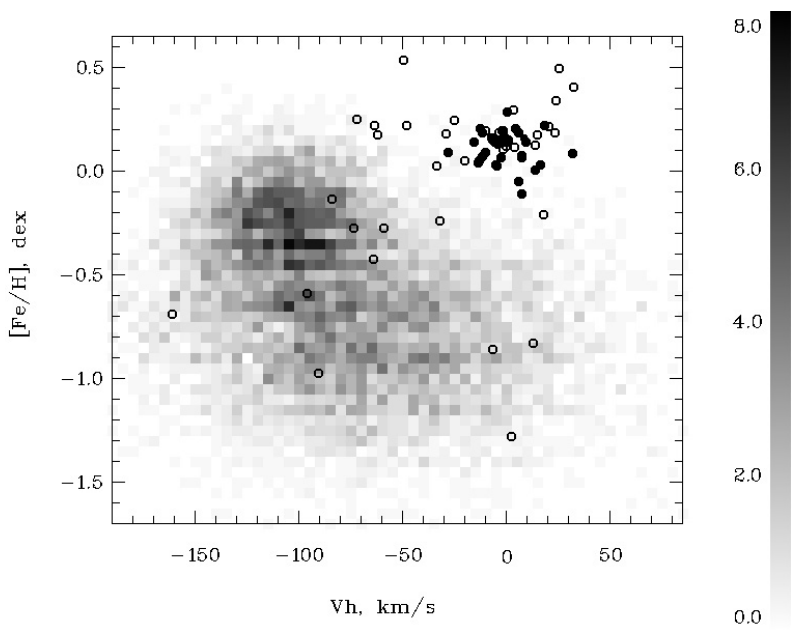

Fig. 1. Distribution in metallicity and heliocentric velocity (Table A.1) of all the stars observed spectroscopically (dots), compared with the density distribution of the same parameters obtained using the Besançon model. Black dots show the stars classified as belonging to BH176.

Figure 1 shows that this region of the Galaxy contains a negligibly small number of stars with metallicities and radial velocities similar to those of member stars of BH176. This and the atmospheric parameters summarised in Table A.1 allow us to identify the stars belonging to the $S C$. These are the criteria: $1.8 \geq V-I \geq 5 \mathrm{mag}, 13.87 \geq I \geq 17.1, V_{\mathrm{h}} \geq-40 \mathrm{~km} \mathrm{~s}^{-1}$, $[\mathrm{Fe} / \mathrm{H}] \geq-0.24$ dex, $3100 \geq T_{\text {eff }} \geq 4900 \mathrm{~K}$, and $-0.5 \geq$ $\log g \geq 3.5$.

\subsection{A representative spectrum of $\mathrm{RC}$ stars in $\mathrm{BH} 176$}

The range of parameters for the RC stars is slightly narrower: $0.8 \geq(V-I)_{0} \geq 1.6,-1.03 \leq M_{I} \leq 0.07,-32 \geq V_{\mathrm{h}} \geq 32$, $-0.24 \geq[\mathrm{Fe} / \mathrm{H}] \geq 0.24,3900 \geq T_{\text {eff }} \geq 4900$, and $2.2 \geq$ $\log g \geq 3.5$. It was important in our analysis to select a sample of stars in BH176 with similar physical parameters for producing a composite spectrum. It was stressed by D11 and in the Introduction of this paper that only RC stars represent a statistically significant sample of bright objects with well-defined photometric parameters. They are not only good distance benchmarks, but also indicators of the evolutionary status of BH176. For comparison, Paczyński \& Stanek (1998) selected RC stars from the HIPPARCOS catalogue (ESA 1997) according to the following photometric parameters: $0.8 \geq(V-I)_{0} \geq 1.25$ and $-1.4 \leq M_{I} \leq 1.1$. According to Girardi \& Salars (2001), the luminosities of such stars in the $V$ and $I$ bands are sensitive to the ages and metallicities of the stellar ensembles they belong to, and also to population effects, i.e. to the element abundance anomalies existing in these ensembles.

A summed spectrum with a high $\mathrm{S} / \mathrm{N}$ can be used to derive more accurate radial velocities and atmospheric parameters for the SC. In order to estimate the stability of the determined characteristics we varied the number of co-added objects. We obtain the following typical accuracies for the atmospheric parameters by comparing the data for stars observed twice (slit $28 \mathrm{a}=$ slit $32 \mathrm{~b}$ ), or objects common with the list of Frinchaboy et al. (2006) $($ slit $37 \mathrm{a}=\mathrm{F} 4190$, slit $18 \mathrm{a}=\mathrm{F} 9312$, slit $21 \mathrm{a}=\mathrm{F} 825): \Delta[\mathrm{Fe} / \mathrm{H}]=$ $0.1 \div 0.2$ dex, $\Delta T_{\text {eff }}=30 \div 50 \mathrm{~K}, \Delta \log (g)=0.1 \div 0.2$ dex.

The mean values for the $31 \mathrm{RC}$ member stars within $\sim 3^{\prime}$ form the centre of BH176 (Table A.1) are the following: $V_{\mathrm{h}}=$ $0 \pm 15 \mathrm{~km} \mathrm{~s}^{-1},[\mathrm{Fe} / \mathrm{H}]=0.1 \pm 0.1 \mathrm{dex}, T_{\text {eff }}=4500 \pm 150 \mathrm{~K}$,
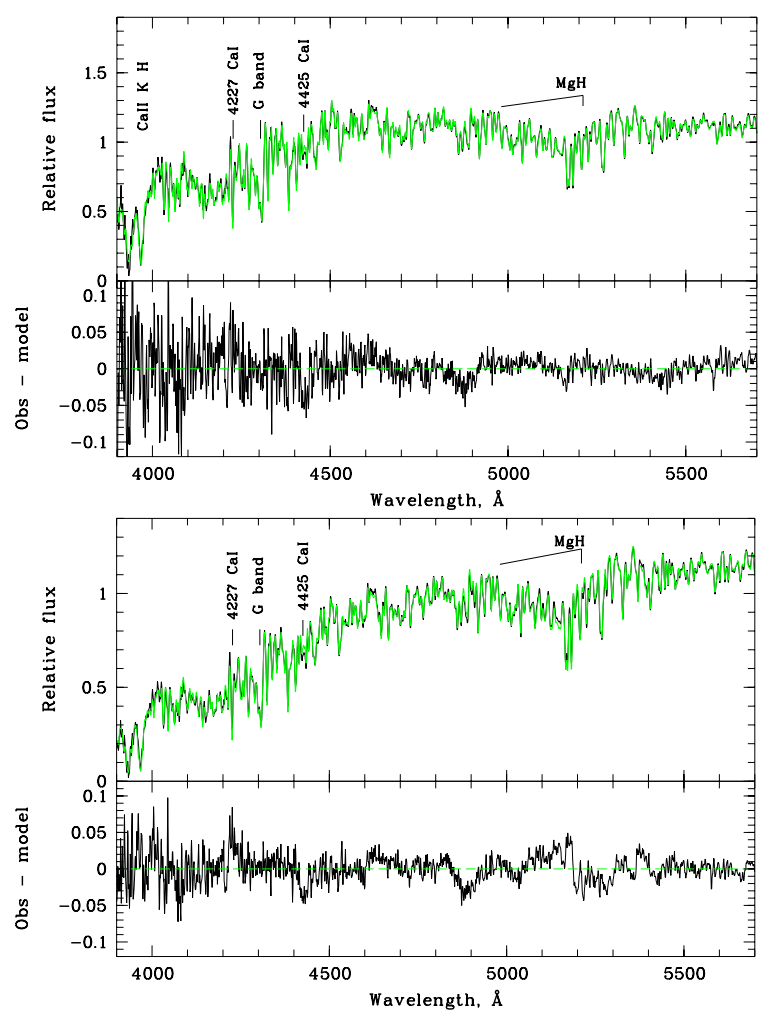

Fig. 2. Top: fitting a representative summed spectrum of RC stars (black) using the ULYSS program and ELODIE 3.2 spectral library. The model spectrum is shown in grey (green in the electronic version). Bottom: comparison of the spectrum of RC stars (black) with the one of HD 184406 from the MILES library (green in the electronic version). The differences between the object and model spectra are shown in the lower panels, where the dotted lines indicate zero differences.

$\log g=2.5 \pm 0.25 \mathrm{~cm} / \mathrm{s}^{2}$. The following parameters were obtained by fitting the co-added RC spectrum (Fig. 2, upper panel) with the ULYSS program and the ELODIE 3.2 stellar library: $V_{\mathrm{h}}=4.4 \pm 0.5 \mathrm{~km} \mathrm{~s}^{-1},[\mathrm{Fe} / \mathrm{H}]=0.1 \pm 0.01 \mathrm{dex}$, $T_{\text {eff }}=4494 \pm 6 \mathrm{~K}$, and $\log g=2.56 \pm 0.02 \mathrm{~cm} / \mathrm{s}^{2}$. The location of all the observed stars and the member ones on the CMD is given in Sect. 5.

\subsection{Abundance analysis using stellar atmosphere models}

We used $T_{\text {eff }}, \log g$, and $[\mathrm{Fe} / \mathrm{H}]$ derived in the previous section for a representative spectrum of RC stars to generate synthetic spectra using the Castelli \& Kurucz (2004) stellar atmosphere models and a software package originally published by Shimansky et al. (2003). Elemental abundance determination at medium resolution is not a new technique (e.g. Kirby et al. 2008, and references therein). The atmospheric parameters and abundances can be adjusted in pixel-to-pixel spectral fits of many metal absorption lines over a wide spectral range. Because our spectrum is of medium resolution, we derived approximate abundances only for the chemical elements influencing the whole spectrum $(\mathrm{Fe})$, a large part of it $(\mathrm{Mg}$, $\mathrm{Ca}, \mathrm{C}$ ) or having several strong dominant lines (see Table 2). For example, the blend of $\mathrm{Mn}(\lambda \sim 4033 \AA)$ (Fig. A.2) includes about twenty strong MnI lines. The method was tested by comparing a high-resolution spectrum of Arcturus with the computed synthetic spectrum (Fig. A.1). The elemental abundances and atmospheric parameters for Arcturus were taken from Ramírez \& Prieto (2011). We extracted the high $\mathrm{S} / \mathrm{N}$ spectrum of Arcturus 
Table 2. Elemental abundances and their uncertainties for the summed spectra of $31 \mathrm{RC}$ stars in BH176 derived in this work using the stellar atmosphere models and literature abundances of a similar star HD 184406.

\begin{tabular}{|c|c|c|c|c|c|c|c|c|c|}
\hline Object & $\begin{array}{c}{[\mathrm{C} / \mathrm{Fe}]} \\
(\mathrm{dex})\end{array}$ & $\begin{array}{c}{[\mathrm{N} / \mathrm{Fe}]} \\
(\mathrm{dex})\end{array}$ & $\begin{array}{c}{[\mathrm{Mg} / \mathrm{Fe}]} \\
(\mathrm{dex})\end{array}$ & $\begin{array}{c}{[\mathrm{Ca} / \mathrm{Fe}]} \\
(\mathrm{dex})\end{array}$ & $\begin{array}{c}{[\mathrm{Ti} / \mathrm{Fe}]} \\
(\mathrm{dex})\end{array}$ & $\begin{array}{c}{[\mathrm{Cr} / \mathrm{Fe}]} \\
(\mathrm{dex})\end{array}$ & $\begin{array}{c}{[\mathrm{Mn} / \mathrm{Fe}]} \\
(\mathrm{dex})\end{array}$ & $\begin{array}{c}{[\mathrm{Co} / \mathrm{Fe}]} \\
(\mathrm{dex})\end{array}$ & $\begin{array}{c}{[\mathrm{Ni} / \mathrm{Fe}]} \\
(\mathrm{dex})\end{array}$ \\
\hline$\overline{\mathrm{BH} 176}$ & 0.15 & 0.15 & 0.25 & 0.25 & 0.35 & 0.25 & -0.05 & 0.25 & -0.05 \\
\hline & \pm 0.15 & \pm 0.20 & \pm 0.10 & \pm 0.15 & \pm 0.20 & \pm 0.20 & \pm 0.20 & \pm 0.20 & \pm 0.20 \\
\hline HD 184406 & $0.22^{1}$ & $0.03^{1}$ & $0.35^{1}$ & $0.13^{2}\left(-0.49^{1}\right)$ & $-0.16^{2}\left(-0.27^{1}\right)$ & $-0.14^{1}$ & - & $0.11^{1}$ & $-0.21^{2}\left(0.26^{1}\right)$ \\
\hline
\end{tabular}

Notes. The superscripts refer to (1) Luck \& Challener (1995) ; (2) McWilliam (1990).

$(S / N>500)$ from the ELODIE library ${ }^{5}$. The description of the method and the list of used lines and atomic constants (oscillator strengths, damping of wings) are given in Appendix A.

We fitted the pseudo-continuum in ULYSS. We thus transformed the shape and mean intensity in the observed spectrum to fit the theoretical one. When using this procedure, it is important that the calculation of the global spectral curvature be performed exactly in the same way for the observed and synthetic spectra, smoothed to the observational resolution. The observed data should have high $\mathrm{S} / \mathrm{N}$, and they should be cleaned and reduced properly prior to determining the pseudo-continuum.

The comparison between the model and the normalised observed spectrum is shown in Fig. A.2 for the most prominent spectroscopic features and in Fig. A.3 for the whole spectrum. One notices a strict repeatability of strong absorption line features consisting of individual lines and line blends in the model and observed spectra. The theoretical spectrum generated using $[\mathrm{Fe} / \mathrm{H}]=-0.15 \mathrm{dex}, \xi_{\text {turb }}=1.6 \mathrm{~km} \mathrm{~s}^{-1}$ and the atmospheric parameters $T_{\text {eff }}=4494 \mathrm{~K}$ and $\log g=2.56$ matches the RC summed spectrum well with an accuracy better than $2 \%{ }^{6}$. The chemical compositions and their uncertainties are summarised in Table 2. According to these data the $\alpha$-element abundance (mean of $[\mathrm{Mg} / \mathrm{Fe}]$ and $[\mathrm{Ca} / \mathrm{Fe}]$ ) is $[\alpha / \mathrm{Fe}]=0.25 \pm 0.07 \mathrm{dex}$.

\subsection{Comparison with objects in stellar libraries}

We used the derived atmospheric parameters and $[\alpha / \mathrm{Fe}]$ to find analogues of our composite spectrum of RC stars in BH176 in the MILES (Sánchez-Blázquez et al. 2006; Cenarro et al. 2007) and ELODIE (Prugniel \& Soubiran 2001; Prugniel et al. 2007) libraries. They both have an optimal coverage of the HR diagram. Nevertheless, the ELODIE coverage is not optimal for RGB stars with metallicities $-0.4>[\mathrm{Fe} / \mathrm{H}]>0.2$ dex (Fig. 1 in Katz et al. 2011; Coelho 2009). In comparison to ELODIE, MILES represents a richer collection of giant stars with known atmospheric parameters and $[\mathrm{Mg} / \mathrm{Fe}]$ ratios. Milone (2011) determined $\mathrm{Mg}$ abundances for a large number of its objects. The coverage in atmospheric parameters and chemical patterns of MILES is large. The library serves as a starting point for stellar population models (Vazdekis et al. 2010) and for the development of theoretical stellar libraries with $\alpha$-element enhanced mixtures (see Coelho 2014, and references therein).

We found only one star that is similar to our object in all parameters. This is the variable star HD 184406 (19:34:05.354 +07:22:44.18 (J2000.0)) with $T_{\text {eff }}=4450 \mathrm{~K}, \log g=2.47$, $[\mathrm{Fe} / \mathrm{H}]=-0.13$ dex, and $\xi_{\text {turb }}=1.8$ (McWilliam 1990). The comparison of its spectrum with the composite spectrum of $\mathrm{RC}$ stars is shown in the lower panel of Fig. 2. We used a

\footnotetext{
5 http://atlas.obs-hp. fr/elodie/

6 This means that the difference between the normalised spectra is $\leq 0.02$.
}

high-resolution high $S / N \sim 90$ spectrum of HD 184406 from the MILES database ${ }^{7}$. The comparison was done in ULYSS. Figure 2 shows that the difference between our spectrum and the reference spectrum does not exceed $5 \%$. The abundances of chemical elements for HD 184406 determined using the highresolution spectra by Luck \& Challener (1995) and McWilliam (1990) are listed in Table 2 for comparison with the approximate data for our representative medium-resolution spectrum. McWilliam (1990) calibrated $T_{\text {eff }}$ using the Johnson filters and estimated the surface gravities from the stellar luminosities and temperatures. We used $[\mathrm{C} / \mathrm{Fe}]$ and $[\mathrm{N} / \mathrm{Fe}]$ estimated for physical gravities. We calculated $[\mathrm{El} / \mathrm{Fe}]$ from $[\mathrm{M} / \mathrm{H}]$ values in Luck \& Challener (1995, from Tables 11 and 6 in that paper). If there were abundances determined for the same element from different lines, we averaged these estimates. These authors derived $[\mathrm{Fe} / \mathrm{H}]=-0.05, T_{\text {eff }}=4375 \mathrm{~K}$ and $\log g=2.65$ for HD 184406 , where $T_{\text {eff }}$ was determined using DDO, Geneva, and Johnson filters. McWilliam (1990) provided $[\mathrm{Fe} / \mathrm{H}]$ relative to the Sun and logarithmic numerical densities of atoms. We calculated $[\mathrm{El} / \mathrm{Fe}]$ abundances from these data taking the atomic data for the Sun from Grevesse (1984) into account as done by that author.

Table 2 shows that the $[\mathrm{Fe} / \mathrm{H}]$ values and the abundances of $\mathrm{C}, \mathrm{N}, \mathrm{Mg}, \mathrm{Ca}$, and $\mathrm{Co}$ of the two objects agree well. There are large differences in the literature estimates for $\mathrm{Ca}$, $\mathrm{Ti}$, and Ni. The Lick indices (Worthey 1994; Worthey \& Ottaviani 1997) measured in the spectra of our object and of HD 184406, smoothed to the resolution of $F W H M=5 \AA$, are in excellent agreement (Table A.3).

\subsection{Three M-type giants}

The knowledge of the radial velocities and physical properties for the three brightest and reddest stars located within $1.5^{\prime}$ from the centre of the SC (slits 12a, 11b, and 9b in Table A.1) is critical for understanding the physical parameters and the nature of BH176. Unfortunately, we did not manage to estimate these data exhaustively using the ELODIE3.2 interpolating arrow $^{8}$ because the spectral library contains very few such objects. The fitting results are listed in Table A.1. The quality of the fits was poor, especially in the case of the reddest star $9 \mathrm{~b}$. To better constrain the properties of the three stars, we inspected the existing spectral libraries for the presence of giant cool stars with similar colours and luminosities (Table 3). We also used the Atlas of digital spectra of cool stars (Turnshek et al. 1985) to constrain the spectral class of each star by visual comparison.

All the selected candidate analogues were M-type giants with luminosity class III. Such cool giant stars are fairly rare

\footnotetext{
7 http://miles.iac.es/pages/stellar-libraries/ miles-library.php

8 http://www.obs.u-bordeaux $1 . \mathrm{fr} / \mathrm{m} 2 \mathrm{a} /$ soubiran/ elodie\$_-\$library.html
} 
Table 3. Colours and heliocentric radial velocities for the three M-type stars and literature data for their analogues.

\begin{tabular}{|c|c|c|c|c|c|c|c|c|c|c|c|c|}
\hline Slit & $\begin{array}{l}V-K \\
(\mathrm{mag})\end{array}$ & $\begin{array}{l}J-K \\
(\mathrm{mag})\end{array}$ & $\begin{array}{c}V_{\mathrm{h}} \\
\left(\mathrm{km} \mathrm{s}^{-1}\right)\end{array}$ & Lit. analogue & $\begin{array}{l}V-K \\
(\mathrm{mag})\end{array}$ & $\begin{array}{l}J-K \\
(\mathrm{mag})\end{array}$ & $\begin{array}{l}T_{\text {eff }} \\
(\mathrm{K})\end{array}$ & $\begin{array}{r}\log (g) \\
\left(\mathrm{cm} \mathrm{s}^{-2}\right)\end{array}$ & $\begin{array}{c}{[\mathrm{Fe} / \mathrm{H}]} \\
(\mathrm{dex})\end{array}$ & $\begin{array}{c}V \text { ampl. } \\
\text { (mag) }\end{array}$ & $\begin{array}{r}\text { Period } \\
\text { (d) }\end{array}$ & Source \\
\hline $12 \mathrm{a}$ & 5.4 & 1.07 & 30 & HD 101153 & 5.450 & 1.16 & 3452 & 0.80 & -0.08 & 0.28 & 30 & 1 \\
\hline $11 \mathrm{~b}$ & 6.9 & 1.29 & 18 & HD 169931 & 5.935 & 1.08 & 3106 & -0.47 & -0.47 & 0.30 & 129 & 2 \\
\hline $9 b$ & 8.6 & 1.32 & 8 & HD 126327 & 9.849 & 1.26 & 3100 & 1.98 & -0.45 & 0.20 & 157 & 2 \\
\hline
\end{tabular}

References. (1) Percy et al. (2001); (2) Lebzelter \& Hinkle (2002).

in our Galaxy and, in particular, in the region of the SC, judging from the Swope spectroscopy of several hundred $M$ giant candidates selected from the 2MASS photometry by Majewski et al. (2004). The Besançon model (see Sect. 3.1) demonstrates that only $\sim 0.1 \%$ stars with the same colours in this area may be giants at the same distance as BH176.

It appears that there are indeed only a few dozen M5III M8III stars in the SIMBAD database ${ }^{9}$ for which all the characteristics (colours, effective temperatures, surface gravities, and metallicities) are known. Spectra of these stars are available in the ELODIE and MILES stellar libraries.

We found only one analogue for each of the stars $11 b$ and $9 b$ (Table 3). For slit 12a we found five stars with similar colours and a full set of data from the literature. The parameters for these five objects are in the range $[\mathrm{Fe} / \mathrm{H}] \sim-0.02 \div-0.34$, $T_{\text {eff }} \sim 3181 \div 3452 \mathrm{~K}$ and $\log (g) \sim 0.47 \div 0.8$. The data for three Galactic field $\mathrm{M}$ giants are given in Table 3, together with the velocities and colours of our three $M$ stars. The columns contain the following information: (1) slit number; $(2,3) V-K$, $J-K$ colour; (4) heliocentric radial velocity, measured with respect to the reference stars; (5) reference M-giant star; $(6,7)$ its $V-K$ and $J-K$ colours; (8) effective temperature; (9) surface gravity; (10) $[\mathrm{Fe} / \mathrm{H}]$; (11) amplitude of the visual variability; (12) period of variability; (13) literature source for the last two columns. The comparison between the observed and literature spectra are shown in Fig. 3. All three Galactic field Mstars appear to be giant semi-regular variables (SRb) with an amplitude of variability $\sim 0.3 \mathrm{mag}$ and variability periods of 30 to 160 days. The calculated heliocentric radial velocities allow us to conclude that our three stars belong to BH176. The velocities are higher than zero unlike the majority of surrounding Galactic stars (Fig. 1). The $T_{\text {eff }}$ and $\log (g)$ of the three stars allow us to classify them as semi-regular variable giants M5III-M8III close to the tip of the RGB.

\section{Proper motions}

The proper motions (PMs) in right ascension $\mu_{\alpha} \cos (\delta)$ and declination $\mu_{\delta}$ extracted for the program stars from the PPMXL catalogue (Roeser et al. 2010) are listed in Table A.1 in mas $\mathrm{yr}^{-1}$. To identify the stars we used the on-line Aladin sky atlas ${ }^{10}$ and coordinate transformations from the positions of the slits in the Gemini image (Sect. 2.1) in equatorial coordinates for epoch 2011.1 to the coordinate system of the PPMXL catalogue. The accuracy of the latter is $\sim 80$ mas relative to the HIPPARCOS reference frame for stars with $K<14$ (Roeser et al. 2010). To derive the transformation equations, we applied the CCMAP and CCTRAN routines in IRAF to five bright relatively isolated stars with $K<12 \mathrm{mag}$. The solution was obtained

\footnotetext{
9 http://simbad.u-strasbg.fr/simbad/

${ }^{10}$ http://aladin.u-strasbg.fr
}
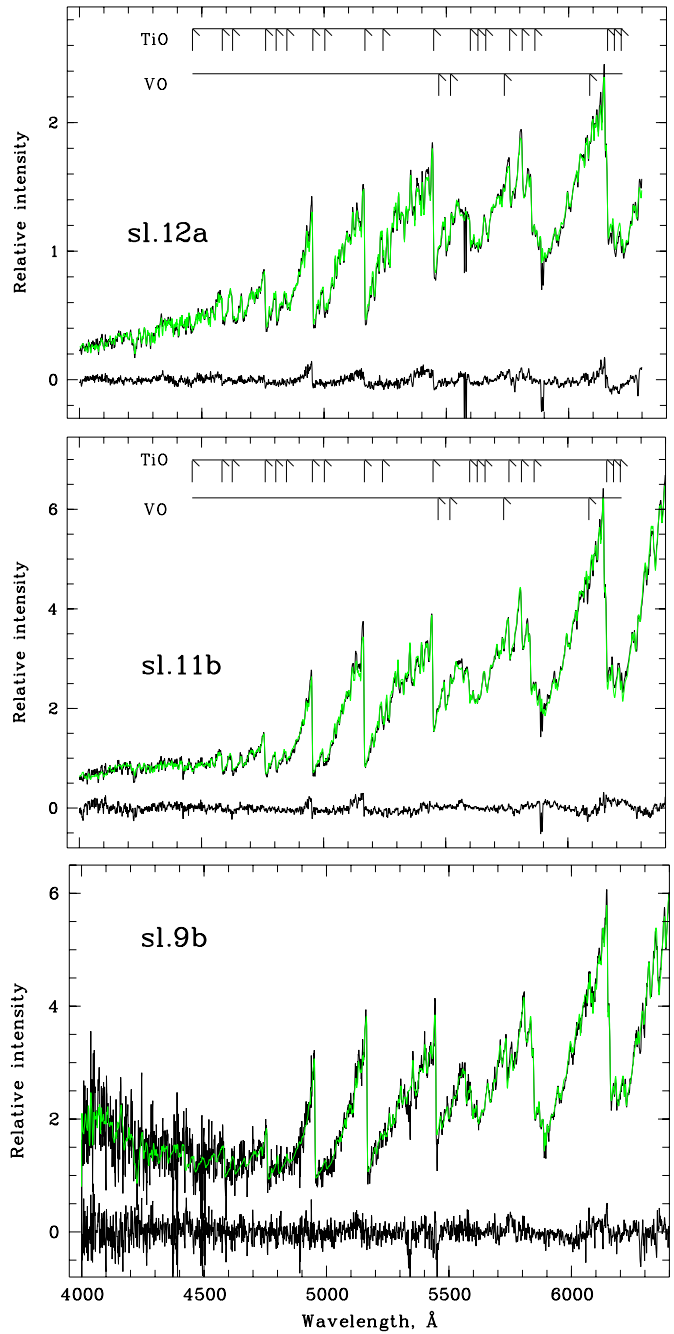

Fig. 3. Spectra of the three M-giant stars (black), slit 12a, slit 11b, and slit 9b, fitted with spectra of HD 101153, HD 169931, and HD 126327, respectively (green in the electronic version). The differences between the object and model spectra are shown at the bottom. The molecular bands of titanium oxide and vanadium oxide are indicated. The derived parameters are listed in Table 3.

with accuracies of 0.002 and $0.037^{\prime \prime}$ for the right ascension and declination, respectively. The accuracy of the solution allowed us to calculate PMs for some of the detected stars by comparison of the coordinates of stars in the PPMXL catalogue, in the VLT, and in the Gemini images. According to the mean $\mu_{\alpha} \cos (\delta)=7 \pm 10 \mathrm{mas} / \mathrm{yr}$ and $\mu_{\delta}=-0.4 \pm 9 \mathrm{mas} / \mathrm{yr}, \mathrm{BH} 176$ is a system unlikely to belong to the Sagittarius stream (Law \& Majewski 2010). 


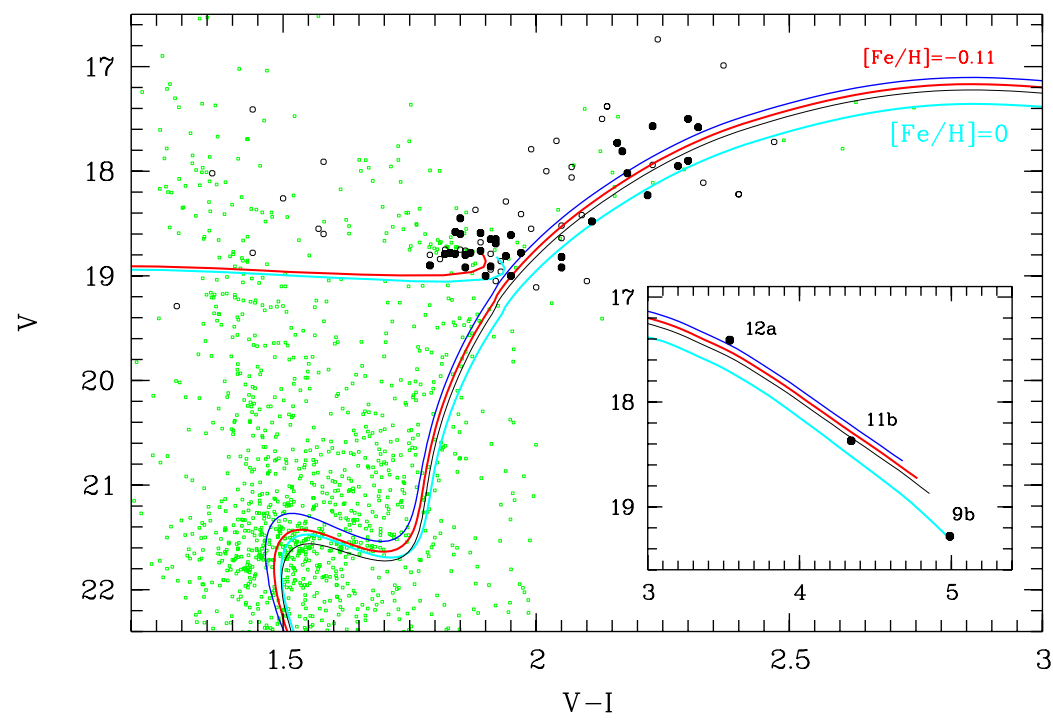

Fig. 4. CMD for stars within $1.5^{\prime}$ from the centre of BH176 (D11; small grey dots). Black open squares indicate the objects observed spectroscopically. Black filled squares show the RC and RGB stars selected according to our criteria to produce the co-added spectra. Alpha-element-enhanced $([\alpha / \mathrm{Fe}]=0.3)$ isochrones computed from the VictoriaRegina stellar models of VandenBerg et al. (2006) are over-plotted: (from top to bottom) $[\mathrm{Fe} / \mathrm{H}]=-0.1 \mathrm{dex}$ and Age $=6$ Gyr, 7 Gyr (red in the electronic version), 8 Gyr; $[\mathrm{Fe} / \mathrm{H}]=0.0$ dex, Age $=7 \mathrm{Gyr}($ cyan in the electronic version).

\section{CMD analysis}

Using the metallicity and $\alpha$-element enhancement for the $\mathrm{RC}$ stars, $[\mathrm{Fe} / \mathrm{H}]=-0.15 \pm 0.1 \mathrm{dex}$, and $[\alpha / \mathrm{Fe}]=0.28 \mathrm{dex}$, obtained in Sect. 3, and the photometric results of D11, we have re-estimated the age, distance from the Sun, and Galactic extinction for BH176 by fitting the CMD with isochrones computed from the Victoria-Regina stellar models of VandenBerg et al. (2006). We used the isochrone corresponding to $[\mathrm{Fe} / \mathrm{H}]=$ $-0.1 \mathrm{dex}$ and $[\alpha / \mathrm{Fe}]=0.3$. The photometric data and the models are shown in Fig. 4. The estimated parameters for BH176 are the following: age $7 \pm 0.5 \mathrm{Gyr}$, distance modulus $(m-M)_{0}=$ $15.91 \pm 0.03 \mathrm{mag}$ (distance from the Sun $15.2 \pm 0.2 \mathrm{kpc}$ ), colour excess $E(V-I)=0.79 \pm 0.03$. The agreement with the results of D11 is very good everywhere. Figure 4 shows that the isochrone corresponding to the derived metallicity, age, and $[\alpha / \mathrm{Fe}]$ fits the observed CMD particularly well. The inset shows the red part of the CMD with the reddest RGB stars. The scatter of the RGB and RC stars agrees with the artificial star photometric results in $\mathrm{D} 11$. The isochrone $[\alpha / \mathrm{Fe}]=0.3 \mathrm{dex},[\mathrm{Fe} / \mathrm{H}]=0.0 \mathrm{dex}$ is slightly off the mean locus of the RGB. Only the TRGB star $9 \mathrm{~b}$ corresponds to it "ideally". What might be the reason? First, this star is fainter than the two neighbours. So, its photometric uncertainty the $V$-band is larger. Second, it is probable that this star is variable. Variability is a common property of SRb stars (see Table 3). Finally, it may have chemical anomalies.

The error budget mostly includes a random component consisting of the uncertainties due to a variable extinction at low Galactic latitudes and of photometric errors. The main source of photometric errors is crowding in dense stellar fields, followed by uncertainties in determining the background level. The artificial star experiments (D11) have reduced the influence not only of the random errors on the results, but also of the systematic ones. The systematic difference between the intrinsic and observed colours and magnitudes includes unknown aperture corrections in dense stellar fields and possible systematic shifts in the photometric zeropoints owing to unexpected changes in the observing conditions. The last component may only be identified by comparing different observations of the same object. We compared the $V$ magnitudes for the three RGB stars (12a, 11b and 9b) using the Gemini and the VLT images and found that $9 \mathrm{~b}$ is brighter by $\sim 0.1 \mathrm{mag}$ in the Gemini frame. Transformations to the Johnson-Cousins system were made using the equation of Fukugita et al. (1996). Including the spectroscopic methods in the analysis allowed us to avoid the systematic differences between the observed and model colours and magnitudes due to unknown helium and light-element contents (see e.g. Salaris 2012, for a detailed discussion of these issues).

\section{Discussion of the nature of $\mathrm{BH} 176$}

BH176 is situated at a large Galactocentric radius: $R_{\mathrm{gc}}=9.4 \mathrm{kpc}$. Its distance components in kpc relative to the Galactic centre are $X_{\mathrm{gc}}=-4.9, Y_{\mathrm{gc}}=-7.9$, and $Z_{\mathrm{gc}}=1.15$. It is located in the outer disk according to our present-day knowledge about the structure of the Galaxy (Gaensler et al. 2008; Moni Bidin et al. 2012; Adibekyan et al. 2012; Haywood et al. 2013, and references therein). The properties of stellar populations in this area are not well known because of its remoteness and the enormous interstellar extinction near the Galactic plane.

It cannot be a member of the Galactic anticentre stellar structure or of the Sagittarius dwarf spheroidal galaxy, because of its too high metallicity (Frinchaboy et al. 2006; Layden \& Sarajedini 2000). BH176 is not an ordinary GC, even though it is away from the Galactic plane and massive $\left(M_{V}=-4.2 \mathrm{mag}\right.$, Radius $=13.2 \mathrm{pc})$. Galactic GCs with metallicities $[\mathrm{Fe} / \mathrm{H}] \sim$ 0 dex are very rare (Harris 1996). Most of them are in the bulge. The closest GC analogue of BH176 is probably Palomar 10 with $R_{\mathrm{gc}}=6.4 \mathrm{kpc}$ and distance components in kpc: $X_{\mathrm{gc}}=4.4$, $Y_{\mathrm{gc}}=4.7, Z_{\mathrm{gc}}=0.3$ (Harris 1996). Its metallicity is $[\mathrm{Fe} / \mathrm{H}] \sim$ -0.1 dex on the Zinn-West metallicity scale (Kaisler et al. 1997) and $M_{V}=-5.8 \mathrm{mag}$. The origin of Palomar 10 has not been firmly established.

BH176 is too metal-rich to be one of the OCs associated with the outer Galactic disk (Frinchaboy et al. 2006; Frinchaboy \& Majewski 2008; Villanova et al. 2010). The mean metallicity of the outer-disk OCs and field stars is $[\mathrm{Fe} / \mathrm{H}] \sim-0.48 \pm 0.12 \mathrm{dex}$ (Bensby et al. 2011; Jacobson et al. 2011), and $[\alpha / \mathrm{Fe}] \sim$ -0.2 dex (Lepine et al. 2014). The metallicity and age of BH176 is similar to that of the thin-disk field stars, but the $\alpha$ element content is higher than for the thick disk component with $[\mathrm{Fe} / \mathrm{H}] \sim 0$ (see e.g. Figs. 7-9 in Haywood et al. 2013). Only a significant contribution of very massive stars that end their lives as SNII can give rise to such an enrichment. However, if the thick disk finished forming stars $\sim 8$ Gyr ago (Haywood et al. 2013), the gas density in this area $\sim 7$ Gyr ago was not as high as in the thin disk, where OCs were effectively destroyed. 
We selected OCs having ages greater than 4 Gyr from the catalogue of Gozha, Borkova, and Marsakov (2012, hereafter: GBM12). Table A.4 summarises the data for the resulting sixteen objects. The successive columns list the following information: (1) OC name; (2) absolute visual magnitude, corrected for Galactic extinction; (3) colour excess; (4) age; (5) $[\mathrm{Fe} / \mathrm{H}]$; (6) $[\mathrm{Mg} / \mathrm{Fe}]$; (7) Galactocentric radius; (8) heliocentric distance towards the north Galactic pole; (9) distance from the Sun. The data in Cols. 1 and 2 were calculated by us using the SIMBAD and NASA/IPAC Extragalactic Database (NED). All other data are from Gozha et al. (2012).

The mean characteristics are the following: $[\mathrm{Fe} / \mathrm{H}]=$ $-0.14 \pm 0.28 \mathrm{dex},[\mathrm{Mg} / \mathrm{Fe}]=0.16 \pm 0.1 \mathrm{dex}$, Age $=5.8 \pm$ 1.8 Gyr. These 16 OCs reside at Galactocentric distances in the range $6.6-20.3 \mathrm{kpc}$, and heliocentric distances toward the north Galactic pole from $Z=-2.51 \mathrm{kpc}$ (Berkeley 20) and $-1.77 \mathrm{kpc}$ (Berkeley 75) to $Z=1.1 \mathrm{kpc}$ (NGC 6791) and $1.7 \mathrm{kpc}$ (Saurer 1) (Table A.4). Eleven OCs of the sixteen are located above $|Z| \sim 0.5 \mathrm{kpc}$. Their absolute visual magnitudes are in the range $M_{V_{0}} \sim-4.9 \div-1.1 \mathrm{mag}$. Nine of the selected clusters were classified by GBM12 as belonging to the thick disk. Some of them may belong to the outer disk as well. For example, Carraro et al. (2007) considered Berkeley 25 and 75 to be members of the outer disk. There may be other, probably even younger, analogues of such objects that have $[\mathrm{Fe} / \mathrm{H}] \sim 0$ and high $[\alpha / \mathrm{Fe}]$ and are inclined with respect to the Galactic surface orbits (see e.g. Friel et al. 2010; Glushkova et al. 2009). BH176 is evidently a member of this old OC sample according to its properties.

Another question is what the nature of these presumably thick disk OCs is. That the $\alpha$-element ratios are high, the ages are in a very wide range, and the locations under the Galactic plane are very different may mean that such objects may have originated as embedded massive SCs from the material of the thin disk, perhaps as the result of its dense gas layers interaction with high-velocity clouds or dark mini-haloes. A similar explanation was suggested by GBM12. These objects were not destroyed by the dissipative processes in the Galactic disk, because their orbits were strongly inclined with respect to its surface.

Part of these clusters might have appeared because of the interaction of the thin disk with gas-rich dwarf satellites moving through it. The recent discovery of vertical waves in the Galactic disk is evidence fof the passage of a small galaxy or dark matter sub-halo (Widrow et al. 2012). There is an extragalactic example of such an interaction. The bright spiral galaxy NGC 6946 located at a distance of $\sim 5.9 \mathrm{Mpc}$ (Karachentsev et al. 2000) has experienced recent powerful star formation bursts revealed by a number of supernovae and star-forming regions.

One of them contains a super star cluster that is as bright as a dwarf galaxy (see Efremov et al. 2007, and references therein). The origin of this object was interpreted by the impact of a highvelocity gas super cloud. Post-impact shock wave collisions are usually associated with high gas pressure, which favours the formation of massive SCs (Elmegreen \& Efremov 1997).

\section{Summary}

Medium-resolution observations of stars in BH176 using the Gemini South Multi-Object Spectrograph, combined with deep VLT photometry, allowed us to determine the distance, chemical composition, and evolutionary status of this outer-disk SC more accurately. The candidate stars for spectroscopic observation were selected using the photometric results of D11. Thirty-one RC stars were identified in BH176 with radial velocities, photometric, and atmospheric parameters in the range
$1.8 \geq V-I \geq 2.4,16 . \geq I \geq 17.1,-32 \geq V_{\mathrm{h}} \geq 32,-0.24 \geq$ $[\mathrm{Fe} / \mathrm{H}] \geq 0.24,3900 \geq T_{\text {eff }} \geq 4900,2.2 \geq \log g \geq 3.5$. The identification of RC stars was indispensable for deriving the distance and extinction for the SC correctly, because only these objects are numerous and bright enough in $\mathrm{BH} 176$ to provide a reliable benchmark. Identifying the RC stars with atmospheric parameters in a narrow range was also important for computing a co-added spectrum that was bright and accurate enough to be approximated by the model of one star. Full-spectrum fitting methods were used to estimate radial velocities, metallicities, effective temperatures, and surface gravities for the individual stars. Stellar atmosphere models and comparison with individual stars in the MILES and ELODIE stellar libraries were used to derive the same parameters plus abundances of different elements for a co-added high $\mathrm{S} / \mathrm{N}$ spectrum of RC stars. Finally, the age, distance from the Sun, and Galactic extinction were specified by comparison of the photometric results in the $V$ and $I$ bands with theoretical isochrones. The fundamental parameters of three bright red M-type giant near the TGRB were estimated using literature analogues with similar spectra and broad-band optical and infrared colours.

The properties of BH176 are fully consistent with the ones of Galactic OCs older than 4 Gyr presumably belonging to the thick disk (GBM12). We speculate that BH176 may have originated as a super-star cluster as a consequence of encounter of the forming thin disk with a high-velocity cloud or with a gasrich dwarf galaxy. BH176 is an interesting target for future deep photometric and high-resolution spectroscopic studies with large telescopes.

Acknowledgements. The Gemini data for this paper were obtained under programme GS-2011A-Q-22 of the Argentinian time share. This research made use of the NASA Astrophysics Data System Bibliographic services (ADS), the NASA/IPAC Extragalactic Database (NED) ${ }^{11}$, the SIMBAD database operated at the CDS (Strasbourg, France), and Google. SME acknowledges a grant RFBR14-02-96501-r-ug-a. S.V.V. acknowledges a grant RFBR13-0200351. We thank Vladimir Marsakov for useful discussions, which helped us to improve the paper, and an anonymous referee for comments.

\section{References}

Adibekyan, V. Z., Sousa, S. G., Santos, N. C., et al. 2012, A\&A, 545, A32 Alonso, A., Arribas, S., \& Martínez-Roger, C. 1999, A\&AS 140, 261 Anders, E., \& Grevesse, N. 1989, Geochim. Cosmochim. Acta, 53, 197 Asplund, M., Grevesse, N., Sauval, A. J., \& Scott P. 2009, ARA\&A, 47, 481 Auer, L. H., \& Heasley, J. 1976, ApJ, 205, 165

Bensby, T., Alves-Brito, A., Oey, M. S., Yong, D., \& Melndez, J. 2011, ApJ, 735, L46

Bidin, C. M., Carraro, G., Méndez, R. A., 2012, AJ, 747, 101

Carraro, G., Geisler, D., Villanova, S., Frinchaboy, P. M., \& Majewski, S. R. 2007, A\&A, 476, 217

Castelli, F., \& Kurucz, R. L. 2003, Modeling of Stellar Atmospheres, eds. N. Piskunov et al., Proc. IAU Symp. 210: Poster A20

Cenarro, A. J., Peletier, R. F., Sánchez-Blázquez, P., et al. 2007, MNRAS, 374, 664

Coelho, P. R. T. 2009, Probing Stellar Populations out to the Distant Universe: CEFALU 2008, eds. G. Giobbi et al., AIP Conf. Proc., 1111, 67 Coelho, P. R. T. 2014, MNRAS, 440, 1027

Davoust, E., Sharina, M., \& Donzelli, C. 2011, A\&A, 528, A70 (D11)

Efremov, Yu. N., Afanasiev, V. L., Alfaro, E. J., et al. 2007, MNRAS, 382, 481

Elmegreen, B. G., \& Efremov, Yu. N. 1997, ApJ, 480, 235

ESA, 1997, VizieR Online Data Catalog, I/1239

Friel, E. D., Jacobson, H. R., \& Pilachowski, C. A. 2010, AJ, 139, 1942

Frinchaboy, P. M., \& Majewski, S. R., 2008, AJ, 136, 118

Frinchaboy, P. M., Muñoz, R. R., Phelps, R. L., Majewski, S. R., \& Kunkel, W. E. 2006, AJ, 131, 922

${ }^{11}$ The NASA/IPAC Extragalactic Database (NED) is operated by the Jet Propulsion Laboratory, California Institute of Technology, under contract with the National Aeronautics and Space Administration. 
Fukugita, M., Ichikawa, T., Gunn, J. E.,et al. 1996, AJ, 111, 1748

Gaensler, B. M., Madsen, G. J., Chatterjee, S., \& Mao, S. A. 2008, PASA, 25, 184

Girardi, L., \& Salaris, M. 2001, MNRAS, 323, 109

Glushkova, E. V., Koposov, S. E., Zolotukhin, I. Y., et al. 2010, Astron. Lett. 36,

Gozha, M. L., Borkova, T. V., \& Marsakov, V. A. 2012, Astron. Lett., 38, 506 (GMB12)

Gray, D. F., \& Toner, C. G. 1986, ApJ, 310, 277

Grevesse, N. 1984, Phys. Scr. T, 8, 49

Harris, W. E. 1996, AJ, 112, 1487, http://www.physics,mcmaster.ca/ Globular.html

Haywood, M., Di Matteo, P., Lehnert, M., Katz, D., \& Gómez, A. 2013, A\&A, 560, A109

Husser, T.-O., Wende-von Berg, S., Dreizler, S., et al. 2013, A\&A, 553, A6

Jacobson, H. R., Pilachowski, C. A., \& Friel, E. D. 2011, AJ, 142, 59

Kaisler, D., Harris, W. E., \& McLaughlin, D. E. 1997, PASP, 109, 920

Karachentsev, I. D., Sharina, M. E., \& Huchtmeier, W. K. 2000, A\&A, 362, 544

Katz, D., Soubiran, C., Cayrel, R., et al. 2011, A\&A, 525, A90

Kharchenko, N. V., Piskunov, A. E., Schilbach, E., Roser, S., \& Scholz, R.-D. 2013, A\&A, 558, A53

Kirby, E. N., Guhathakurta, P., \& Sneden, C. 2008, ApJ, 682, 1217

Koleva, M., Prugniel, P., Bouchard, A., \& Wu, Y., 2009, A\&A, 501, 1269

Kruijssen, J. M. Diederik, 2014, in Classical and Quantum Gravity (IOP) for a focus issue on Galactic Centres, eds. Clifford Will, \& Pau Amaro-Seoane, [arXiv: 1407.2953]

Kurucz, R. L., CD-Room, 1994 (Cambridge)

Kurucz, R. L., \& Furenlid I. 1979, Sample Spectral Atlas for Sirius, SAO Spec. Rep., 387

Law, D. R., \& Majewski, S. R. 2010, ApJ, 718, 1128

Layden, A. C., \& Sarajedini, A. 2000, AJ, 119, 1760

Lebzelter, T., \& Hinkle K. H. 2002, A\&A, 393, 563

Lépine, J. R. D., Andrievky, S., Barros, D. A., Junqueira, T. C., \& Scarano, S. 2014, eds. Feltzing S. et al., IAU Symp., 298, 86

Luck, R. E., Challener, S. L., 1995, AJ, 110, 2968

Majewski, S. R., Kunkel, W. E., Law, D. R., et al., 2004, AJ, 128, 245

Milone, A. de C., Sansom A. E., \& Sánchez-Blázquez P. 2011, MNRAS, 414, 1227

McWilliam, A. 1990, ApJS, 74, 1075

Meisner, A. M., Frebel, A., Juric, M., \& Finkbeiner, D. P. 2012, ApJ, 753, 116

Menzhevitski, V. S., Shimanskaya, N. N., Shimansky, V. V., \& Kudryavtsev, D. O. 2014, Astrophys. Bull., 69, 121
Nersisyan, S. E., Shavrina, A. V., \& Yaremchuk, A. A. 1989, Astrofizika, 30 247

Ortolani, S., Bica, E., \& Barbuy, B. 1995, A\&A, 300, 726

Paczyński, B., \& Stanek, K. Z. 1998, ApJ, 494, L219

Percy, J. R., Dunlop, H., Kassim, L., \& Thompson, R. R. 2001, Inform. Bull. Variable Stars, 5041, 1

Phelps, R. L., \& Schick, M. 2003, AJ, 126, 265

Prugniel, P., \& Soubiran, C. 2001, A\&A, 369, 1048

Prugniel, P., Soubiran, C., Koleva, M., \& Le Borgne, D. 2007, VizieR On-line Data Catalog: II/1251

Prugniel, P., Vauglin, I., \& Koleva, M. 2011, A\&A, 531, A165

Ramírez, I., \& Prieto, A. C. 2011, ApJ, 743, 135

Robin, A. C., Reyle, C., Derriere, S., \& Picaud, S. 2003, A\&A, 409, 523

Robin, A. C., Reyle, C., Derriere, S., Picaud, S. 2004, A\&A, 416, 157

Roeser, S., Demleitner, M., \& Schilbach, E. 2010, AJ, 139, 2440

Salaris, M. 2012, ApSS, 341, 65

Sánchez-Blázquez, P., Peletier, R. F., Jiménez-Vicente, J et al., 2006, MNRAS, 371,703

Sakhibullin, N. A., 1983, Trudy Kazan. Gor. Astron. Obs., 48, 9

Shimansky, V. V., Borisov N. V., \& Shimanskaya, N. N. 2003, Astron. Rep., 47 763

Shimanskaya, N. N., Bikmaev I. F., \& Shimansky, V. V. 2011, Astrophys. Bull., 66,332

Smith, M. A., \& Dominy, J. F. 1979, ApJ, 231, 477

Suleimanov, V. F. 1996, Astron. Lett., 22, 92

Turnshek, D. E., Turnshek, D. A., Craine, E. R., \& Boeshaar, P. C. 1985, An Atlas of Digital Spectra of Cool Stars (Tucson: Western Research Company)

Unsöld, A. 1955, Physik der Sternatmospheren, MIT besonderer Berucksichtigung der Sonne (Berlin: Springer)

VandenBerg, D. A., Bergbusch, P. A., \& Dowler, P. D. 2006, ApJS, 162, 375

Van den Bergh, S., \& Hagen, G. L. 1975, AJ, 80, 11

Vazdekis, A., Sánchez-Blázquez, P., Falcón-Barroso, J., et al. 2010, MNRAS, 404, 1639

Vidal, C. R., Cooper, J., \& Smith, E. W. 1973, A\&AS. 25, 37

Villanova, S., Randich, S., Geisler, D., Carraro, G., \& Costa, E. 2010, A\&A, 509, A102

Widrow, L. M., Gardner, S., Yanny, B., Dodelson, S., \& Chen, H.-Y. 2012, ApJ, 750, L41

Worthey, G. 1994, ApJS, 95, 107

Worthey, G., \& Ottaviani, D. L. 1997, ApJS, 111, 377

Wu, Y., Singh, H. P., Prugniel, P., Gupta, R., \& Koleva, M. 2011, A\&A, 525, A71

Pages 10 to 15 are available in the electronic edition of the journal at http://www . aanda.org 


\section{Appendix A: Computation of synthetic spectra using stellar atmosphere models}

To derive the abundances of different chemical elements, we computed synthetic spectra with and without atomic and molecular lines. We used the software package SPECTR (Shimansky et al. 2003; see also Menzhevitski et al. 2014 for its latest version). Shimanskaya et al. (2011) used this program for deriving chemical abundances for the secondary in the close binary system FF Aqr. They show that lines of chemical elements in highresolution spectra strongly broadened by rotation are reproduced well by the theoretical spectra.

Our synthetic spectra are based on plane-parallel, hydrostatic stellar atmosphere models for a given set of parameters $\left(T_{\text {eff }}, \log g,[\mathrm{M} / \mathrm{H}]\right)$ computed by interpolating a model grid of Castelli \& Kurucz (2003) using the technique described by Suleimanov (1996). The solar chemical abundances and isotope compositions were specified using the data of Asplund et al. (2006) for Fe, C, N, and O and of Anders \& Grevesse (1989) for all the other elements.

The radiative transfer equation at each frequency (wavelength) was solved using the Hermite method with the determination of specific radiation intensities for fixed angles. For a model atmosphere, we calculated the fluxes emerging in three basic directions, with inclinations to the surface of $62^{\circ}, 30^{\circ}, 8^{\circ}$. The stellar surface was then subdivided into sectors. The fluxes emerging from sectors towards the observer were derived by interpolating the radiation intensities at the three basic angles to the actual visibility angle.

The computation of synthetic spectra took about 600000 atomic and more than $1800000{ }^{12} \mathrm{CH},{ }^{13} \mathrm{CH}$, and $\mathrm{SiH}$ molecular lines into account from the lists of Kurucz (1994) and Castelli \& Kurucz (2003), and 28 bands of 10 molecules (VO, TiO, SO, SiO, NO, MgO, MgH, CO, CN, AlO) computed in terms of the theory of Nersisyan et al. (1989) and kindly provided by Ya. V. Pavlenko. We used the empirical oscillator strengths from Shimanskaya et al. (2011) for 1350 strong optical lines in the $13900-7000 \AA$ wavelength range.

We computed the profiles of the HI lines according to the broadening theories of Vidal et al. (1973) and Griem (1960). The standard Voigt profiles for the remaining lines were calculated with broadening due to thermal motion and microturbulence, natural damping, Stark broadening in the approximation of Kurucz \& Furenlid (1979), and van der Waals broadening with constants determined from the classical Gray formula (Unsold 1955 ) with scaling factors $\Delta \log C_{6}=0.7 \div 1.2$ (Shimanskaya et al. 2011). The synthetic spectra were binned to the resolution of $0.05 \AA$. We estimated the intensity uncertainties of the line profiles provided by this value to be within $0.005 \%$ of the continuum flux.
We modelled the line profiles $\mathrm{HI}, \mathrm{MgI}, \mathrm{MgII}, \mathrm{AlI}$, and CaII taking deviations from local thermodynamic equilibrium (LTE) into account. For each model atmosphere, the non-LTE populations were computed using the complete linearisation method of Auer \& Heasley (1976) with the package NONLTE3 of Sakhibullin (1983).

Summing the radiation from all the aforementioned sectors of the stellar atmosphere considering their areas and local radial velocities due to stellar rotation and radial and tangential microturbulence yielded the integrated radiation from the atmosphere. The resulting stellar spectra were broadened in accordance with the instrumental function of the spectrograph.

\section{A.1. Determining the chemical abundances}

Since the full width at half maximum in our observed spectra is $F W H M \sim 5 \AA$, almost all spectral lines are weak and blended. The most intense of them are dominant. At this resolution, it is possible to derive the abundance of a chemical element accurately $(\sigma \leq 0.15 \mathrm{dex})$ in spectra with high $\mathrm{S} / \mathrm{N}$ per resolution bin $(S / N \geq 100)$, if this element 1$)$ influences the whole spectrum or a large part of it $(\Delta \lambda \sim 100 \AA)$; and 2) has several strong dominant lines. If an element has just one dominant line and contributes $\geq 50 \%$ of the line intensity, the accuracy of the derived abundance is $\sim 0.2$ dex. The iron abundance and the microturbulent velocity ( $\left.\xi_{\text {turb }}\right)$ influence the whole spectrum. If $\xi_{\text {turb }}$ is incorrect, it is not possible to achieve an optimal agreement between strong and weak iron lines in the theoretical and observed spectra. The macroturbulent and stellar rotation velocities were not taken into account, because these effects are too weak to be detectable at our observational resolution (Smith \& Dominy 1979; Gray \& Toner 1986). The following elements have prominent atomic absorption lines that are dominant at our resolution: MgI $5167 \AA, 5172 \AA$, $5183 \AA$, CaI $4226 \AA$, CaII $3933 \AA$, and CaII $3968 \AA$. The shapes of strong molecular bands, such as $\mathrm{CH}, \mathrm{MgH}$ including hundreds of lines. are easily recognised and reproduced well by the synthetic spectrum.

Figure A. 2 shows theoretical spectra calculated with the stellar atmosphere model corresponding to the following parameters: $[\mathrm{M} / \mathrm{H}]=0.0 \mathrm{dex}, T_{\text {eff }}=4494 \mathrm{~K}$, and $\log g=2.56$. In each of the panels, abundances for one of the elements are varied, while the abundances of other elements have the fixed values listed in Table 2. The derivation of the iron abundance is illustrated in the top right-hand panel. In the course of fitting $[\mathrm{Fe} / \mathrm{H}]$, the micro-turbulent velocity $\xi_{\text {turb }}$ was estimated (see top left panel of Fig. A.2). These two values $\left([\mathrm{Fe} / \mathrm{H}]\right.$ and $\left.\xi_{\text {turb }}\right)$ are closely related. A normalised summed spectrum of $31 \mathrm{RC}$ stars (Table A.1) is shown by a solid line. The fitting of the whole spectrum is illustrated in Fig. A.3. 
Table A.1. Parameters for all stars observed spectroscopically (see Sect. 3.1 for details).

\begin{tabular}{|c|c|c|c|c|c|c|c|c|c|c|c|c|c|c|c|}
\hline Slit & $\begin{array}{c}\text { RA (J2000.0) Dec } \\
\left(\mathrm{h} \mathrm{m} \mathrm{s}^{\circ \prime \prime \prime}\right)\end{array}$ & $\begin{array}{c}I \\
(\mathrm{mag})\end{array}$ & $\begin{array}{l}V-I \\
(\mathrm{mag})\end{array}$ & $\begin{array}{c}K_{\mathrm{s}} \\
(\mathrm{mag})\end{array}$ & $\begin{array}{l}J-K_{\mathrm{s}} \\
(\mathrm{mag})\end{array}$ & $\begin{array}{l}T_{\text {eff }}^{V-K} \\
(\mathrm{~K})\end{array}$ & $\begin{array}{l}\text { Dist. } \\
\left({ }^{\prime \prime}\right)\end{array}$ & $\begin{array}{c}V_{\mathrm{h}} \\
\left(\mathrm{km} \mathrm{s}^{-1}\right)\end{array}$ & $\begin{array}{c}{[\mathrm{Fe} / \mathrm{H}]} \\
(\mathrm{dex})\end{array}$ & $\begin{array}{l}T_{\text {eff }} \\
(\mathrm{K})\end{array}$ & $\begin{array}{c}\log g \\
\left(\mathrm{~cm} / \mathrm{s}^{2}\right)\end{array}$ & $\begin{array}{l}\mu_{\alpha} \cos (\delta) \\
(\mathrm{mas} / \mathrm{yr})\end{array}$ & $\begin{array}{c}\mu_{\delta} \\
(\mathrm{mas} / \mathrm{yr})\end{array}$ & $S / N$ & Note \\
\hline 01a & $15: 39: 23.835-50: 04: 18.53$ & 15.99 & 2.07 & 13.00 & 0.85 & 4051 & 166.0 & $32 \pm 10$ & 0.40 & 4170 & 2.6 & 40 & 29 & 39 & RGB? \\
\hline $02 \mathrm{a}$ & $15: 39: 24.756-50: 03: 31.22$ & 17.02 & 1.58 & & & & 153.1 & $-63 \pm 5$ & 0.22 & 5870 & 4.2 & & & 26 & \\
\hline 04a & $15: 39: 22.960-50: 00: 37.45$ & 16.76 & 1.50 & 14.88 & 0.60 & 5583 & 183.9 & $-62 \pm 5$ & 0.18 & 5639 & 4.5 & & & 30 & \\
\hline $05 \mathrm{a}$ & $15: 39: 20.099-50: 02: 37.23$ & 14.62 & 2.37 & 11.65 & 0.93 & 3900 & 103.3 & $-33 \pm 5$ & 0.02 & 3893 & 1.4 & -7 & -1 & 56 & ${ }^{* *} \mathrm{RGB}, \mathrm{TiO}$ \\
\hline 06a & $15: 39: 20.685-50: 00: 08.75$ & 16.49 & 1.88 & 14.16 & 0.65 & 4568 & 192.7 & $-161 \pm 5$ & -0.68 & 4467 & 2.0 & 4 & 5 & 24 & \\
\hline $07 a$ & $15: 39: 18.376-50: 04: 47.50$ & 16.98 & 1.57 & 14.73 & 0.72 & 4977 & 147.5 & $-9 \pm 15$ & -0.06 & 5100 & 3.5 & & & 28 & $\mathrm{RC}$ ? \\
\hline $08 \mathrm{a}$ & $15: 39: 12.019-50: 02: 47.41$ & 16.60 & 1.85 & & & & 24.9 & $32 \pm 7$ & 0.08 & 4635 & 2.3 & 19 & 11 & 32 & ${ }^{*} \mathrm{RC}$ \\
\hline $09 \mathrm{a}$ & $15: 39: 15.627-50: 02: 36.85$ & 15.97 & 1.44 & & & & 59.8 & $-5 \pm 10$ & 0.03 & 6000 & 4.1 & & & 56 & \\
\hline 10a & $15: 39: 11.242-50: 03: 14.81$ & 16.74 & 1.91 & 14.68 & 0.72 & 4808 & 32.0 & $-32 \pm 13$ & -0.24 & 4852 & 3.5 & & & 34 & ${ }^{*} \mathrm{RC}$ ? \\
\hline 11a & $15: 39: 08.698-50: 03: 04.50$ & 16.74 & 1.84 & & & & 18.5 & $-2 \pm 14$ & 0.15 & 4491 & 2.7 & & & 28 & ${ }^{*} \mathrm{RC}$ \\
\hline $12 \mathrm{a}$ & 15:39:07.829-50:03:10.43 & 13.87 & 3.54 & 10.24 & 1.05 & 3519 & 27.3 & 30: & -0.02 & 3261 & 0.6 & 12 & 8 & 20 & $\mathrm{SRb}$ \\
\hline 13a & $15: 39: 06.999-50: 03: 16.63$ & 16.80 & 1.97 & & & & 37.0 & $18 \pm 15$ & 0.22 & 4596 & 2.6 & -5 & -4 & 23 & ${ }^{*} \mathrm{RC}$ \\
\hline $14 \mathrm{a}$ & $15: 39: 09.249-50: 02: 50.23$ & 16.73 & 1.92 & & & & 2.9 & $-11 \pm 18$ & 0.07 & 4297 & 2.3 & 14 & 1 & 24 & ${ }^{*} \mathrm{RC}$ \\
\hline $15 \mathrm{a}$ & $15: 39: 13.199-50: 03: 35.23$ & 15.34 & 2.23 & 12.50 & 0.84 & 4035 & 59.6 & $23 \pm 12$ & 0.18 & 4019 & 2.1 & 9 & 5 & 44 & ${ }^{* *}$ RGB \\
\hline 16a & 15:39:17.168-50:04:18.90 & 17.13 & 1.92 & 14.53 & 0.75 & 4348 & 118.2 & $24 \pm 18$ & 0.34 & 4428 & 2.6 & & & 20 & $\mathrm{RC} ?$ \\
\hline $17 \mathrm{a}$ & $15: 39: 14.725-50: 00: 41.88$ & 17.11 & 2.00 & 14.65 & 0.61 & 4403 & 136.1 & $5 \pm 12$ & 0.20 & 4409 & 2.7 & & & 17 & ${ }^{*} \mathrm{RC}$ \\
\hline $18 \mathrm{a}$ & $15: 39: 12.595-50: 01: 59.00$ & 15.67 & 2.28 & 12.86 & 0.83 & 4020 & 57.6 & $0 \pm 15$ & 0.12 & 3973 & 1.9 & 5 & -6 & 37 & ${ }^{* *} \mathrm{RGB}, \mathrm{TiO}$ \\
\hline 20a & $15: 39: 16.379-50: 03: 09.73$ & 16.87 & 2.05 & 14.10 & 0.90 & 4166 & 70.3 & $6 \pm 17$ & 0.18 & 4286 & 2.5 & -3 & 3 & 21 & ${ }^{*} \mathrm{RC}$ \\
\hline $21 \mathrm{a}$ & $15: 39: 10.609-50: 02: 27.41$ & 16.91 & 1.87 & & & & 23.4 & $8 \pm 18$ & 0.06 & 4508 & 2.2 & 7 & -14 & 19 & ${ }^{*} \mathrm{RC}$ \\
\hline $22 \mathrm{a}$ & $15: 39: 10.060-50: 02: 22.50$ & 16.91 & 1.86 & & & & 25.5 & $-59 \pm 5$ & -0.28 & 5512 & 4.2 & & & 45 & \\
\hline $23 \mathrm{a}$ & 15:39:17.685-50:01:08.08 & 15.80 & 1.99 & & & & 127.6 & $13 \pm 18$ & -0.83 & 3878 & 4.6 & & & 35 & Mg-rich? \\
\hline $24 a$ & $15: 38: 56.585-50: 05: 06.38$ & 15.82 & 2.40 & 12.93 & 0.90 & 3923 & 186.1 & $20 \pm 12$ & 0.12 & 3966 & 2.2 & 5 & -11 & 34 & RGB? \\
\hline $25 \mathrm{a}$ & $15: 39: 05.899-50: 03: 26.43$ & 16.01 & 2.22 & 13.22 & 0.74 & 4063 & 51.4 & $20 \pm 11$ & 0.21 & 4031 & 2.3 & & & 31 & ${ }^{* *} \mathrm{RGB}$ \\
\hline $26 \mathrm{a}$ & 15:39:03.110-50:03:03.94 & 18.00 & 1.29 & & & & 64.3 & $-96 \pm 9$ & -0.59 & 6006 & 4.4 & & & 24 & \\
\hline $27 \mathrm{a}$ & $15: 39: 00.618-50: 03: 30.10$ & 17.02 & 1.79 & & & & 94.9 & $1 \pm 10$ & 0.28 & 4554 & 2.7 & & & 39 & ${ }^{*} \mathrm{RC}$ \\
\hline $28 \mathrm{a}$ & $15: 38: 58.105-50: 01: 33.20$ & 16.67 & 1.95 & & & & 132.5 & $16 \pm 15$ & 0.02 & 4283 & 2.4 & & & 25 & ${ }^{*} \mathrm{RC}$ \\
\hline $29 a$ & 15:38:57.099-50:02:48.90 & 15.89 & 2.07 & 13.29 & 0.97 & & 119.0 & $15 \pm 11$ & 0.08 & 3955 & 2.2 & 19 & 11 & 34 & $\mathrm{RC} ?$ \\
\hline 30a & $15: 38: 55.819-50: 01: 09.80$ & 15.25 & 2.47 & 12.28 & 0.94 & 3871 & 164.2 & $-10 \pm 7$ & 0.19 & 3822 & 1.4 & 6 & -1 & 39 & ${ }^{* *}$ RGB \\
\hline $31 \mathrm{a}$ & $15: 39: 01.959-50: 03: 43.43$ & 15.37 & 2.13 & 12.49 & 0.89 & 4057 & 91.0 & $21 \pm 5$ & 0.00 & 3950 & 2.2 & & & 51 & $\mathrm{RC} ?$ \\
\hline $32 \mathrm{a}$ & $15: 39: 03.929-50: 02: 51.70$ & 15.84 & 2.18 & 13.11 & 0.87 & 4113 & 53.2 & $7 \pm 19$ & 0.07 & 4086 & 2.0 & 15 & 14 & 35 & ${ }^{* *} \mathrm{RGB}, \mathrm{TiO}$ \\
\hline $33 \mathrm{a}$ & $15: 38: 59.419-50: 01: 52.40$ & 16.88 & 1.91 & & & & 111.5 & $1 \pm 18$ & 0.15 & 4524 & 2.5 & & & 27 & ${ }^{*} \mathrm{RC}$ \\
\hline $34 \mathrm{a}$ & 15:39:04.908-50:01:46.80 & 16.87 & 1.89 & 14.10 & 1.09 & 4258 & 75.5 & $-4 \pm 20$ & 0.02 & 4442 & 2.5 & -4 & -13 & 25 & ${ }^{*} \mathrm{RC}$ \\
\hline $35 \mathrm{a}$ & $15: 39: 06.535-50: 01: 38.41$ & 16.60 & 2.05 & 14.35 & 0.79 & 4497 & 75.1 & $-7 \pm 20$ & -0.86 & 3807 & 4.6 & & & 21 & Mg-rich? \\
\hline $36 \mathrm{a}$ & $15: 39: 02.478-50: 04: 59.10$ & 15.71 & 2.23 & 12.88 & 0.77 & 4037 & 147.7 & $14 \pm 15$ & 0.12 & 4067 & 2.3 & & & 41 & RGB \\
\hline $37 \mathrm{a}$ & 15:38:59.908-50:04:13.70 & 15.24 & 2.14 & & & & 125.8 & $15 \pm 5$ & 0.17 & 4194 & 2.5 & & & 59 & RGB \\
\hline $38 \mathrm{a}$ & $15: 39: 01.375-50: 01: 52.31$ & 16.93 & 1.93 & & & & 95.7 & $-12 \pm 20$ & 0.20 & 4475 & 2.2 & & & 23 & ${ }^{*} \mathrm{RC}$ \\
\hline 01b & $15: 39: 22.310-50: 02: 46.22$ & 15.67 & 2.04 & & & & 123.2 & $-72 \pm 5$ & 0.24 & 4328 & 2.6 & & & 47 & \\
\hline $02 b$ & $15: 39: 25.082-50: 01: 04.15$ & 17.03 & 1.93 & & & & 183.0 & $-13 \pm 18$ & 0.05 & 4476 & 2.1 & & & 20 & ${ }^{*} \mathrm{RC}$ \\
\hline $03 b$ & $15: 39: 20.448-50: 01: 13.30$ & 16.36 & 1.94 & & & & 142.1 & $2 \pm 13$ & -1.28 & 4236 & 1.0 & & & 33 & \\
\hline $04 \mathrm{~b}$ & $15: 39: 19.549-50: 03: 16.51$ & 17.03 & 1.91 & & & & 101.6 & $-4 \pm 19$ & 0.14 & 4587 & 2.4 & & & 22 & ${ }^{*} \mathrm{RC}$ \\
\hline $05 \mathrm{~b}$ & $15: 39: 18.223-50: 03: 39.70$ & 16.66 & 1.36 & & & & 98.8 & $-48 \pm 11$ & 0.22 & 5802 & 4.6 & & & 47 & \\
\hline $06 b$ & $15: 39: 17.452-50: 01: 20.81$ & 14.50 & 2.24 & 11.93 & 1.36 & 4168 & 116.5 & $-20 \pm 5$ & 0.05 & 4036 & 1.8 & & & 72 & RGB,TiO \\
\hline 07b & $15: 39: 11.878-50: 04: 14.78$ & 16.95 & 2.10 & & & & 90.4 & $-84 \pm 18$ & -0.13 & 5819 & 4.4 & & & 43 & \\
\hline $08 \mathrm{~b}$ & $15: 39: 08.309-50: 03: 34.81$ & 16.76 & 1.85 & 14.31 & 0.68 & 4531 & 48.2 & $9 \pm 15$ & 0.13 & 4603 & 2.8 & 1 & 10 & 30 & ${ }^{*} \mathrm{RC}$ \\
\hline $09 b$ & $15: 39: 10.599-50: 03: 03.75$ & 14.30 & 4.99 & 8.89 & 1.31 & & 19.0 & 8: & & & & 20 & 4 & 87 & $\mathrm{SRb}$ \\
\hline $10 \mathrm{~b}$ & $15: 39: 11.258-50: 03: 10.40$ & 16.95 & 1.84 & 14.68 & 0.72 & 4691 & 29.0 & $-2 \pm 20$ & 0.19 & 4545 & 2.4 & -14 & 14 & 24 & ${ }^{*} \mathrm{RC}$ \\
\hline $11 \mathrm{~b}$ & $15: 39: 06.855-50: 02: 53.51$ & 14.03 & 4.34 & 9.70 & 1.28 & & 25.4 & 18: & -0.06 & 3106 & 0.0 & 15 & 4 & 30 & $\mathrm{SRb}$ \\
\hline $12 \mathrm{~b}$ & 15:39:07.409-50:03:35.83 & 16.87 & 1.94 & 14.77 & 0.84 & 4743 & 51.8 & $8 \pm 13$ & -0.11 & 4513 & 2.5 & 22 & 7 & 27 & ${ }^{*} \mathrm{RC}$ \\
\hline $13 b$ & $15: 39: 05.295-50: 02: 43.73$ & 15.60 & 2.30 & 12.73 & 0.84 & 3982 & 40.1 & $4 \pm 11$ & 0.11 & 3999 & 1.7 & 7 & -4 & 41 & ${ }^{* *} \mathrm{RGB}, \mathrm{TiO}$ \\
\hline $14 \mathrm{~b}$ & $15: 39: 06.378-50: 02: 55.30$ & 16.94 & 1.86 & & & & 30.5 & $-16 \pm 17$ & 0.13 & 4580 & 2.4 & & & 21 & ${ }^{*} \mathrm{RC}$ \\
\hline $15 b$ & $15: 39: 09.150-50: 02: 20.70$ & 15.57 & 2.16 & 12.90 & 0.77 & 4160 & 27.1 & $-5 \pm 7$ & 0.14 & 4093 & 2.0 & 4 & -6 & 46 & ${ }^{* *} \mathrm{RGB}, \mathrm{TiO}$ \\
\hline $16 \mathrm{~b}$ & $15: 39: 12.478-50: 04: 15.20$ & 16.57 & 1.99 & 13.99 & 0.66 & 4337 & 92.8 & $4 \pm 11$ & 0.29 & 4242 & 2.7 & & & 29 & $\mathrm{RC}$ ? \\
\hline $17 \mathrm{~b}$ & $15: 39: 14.059-50: 03: 24.86$ & 17.06 & 1.86 & 13.69 & 0.73 & 3964 & 57.8 & $-25 \pm 7$ & 0.24 & 4646 & 2.8 & & & 23 & $\mathrm{RC} ?$ \\
\hline $18 \mathrm{~b}$ & $15: 39: 15.089-50: 03: 08.03$ & 16.34 & 2.09 & 13.72 & 0.70 & 4235 & 58.0 & $-3 \pm 11$ & 0.06 & 4197 & 2.3 & -1 & 4 & 28 & ${ }^{* *} \mathrm{RGB}$ \\
\hline $19 \mathrm{~b}$ & $15: 39: 14.615-50: 02: 20.81$ & 16.38 & 2.11 & 13.72 & 0.91 & 4199 & 56.6 & $-4 \pm 15$ & 0.13 & 4143 & 2.3 & 6 & -4 & 28 & ${ }^{* *} \mathrm{RGB}, \mathrm{TiO}$ \\
\hline $20 \mathrm{~b}$ & $15: 39: 13.592-50: 02: 58.73$ & 17.04 & 1.95 & & & & 41.3 & $0 \pm 17$ & 0.14 & 4470 & 2.3 & -11 & -1 & 21 & ${ }^{*} \mathrm{RC}$ \\
\hline $21 b$ & $15: 39: 05.778-50: 03: 12.70$ & 15.26 & 2.32 & & & & 43.5 & $-10 \pm 13$ & 0.09 & 3864 & 1.8 & & & 40 & ${ }^{* *} \mathrm{RGB}$ \\
\hline $22 b$ & $15: 39: 09.579-50: 02: 50.73$ & 16.77 & 1.92 & 14.32 & 0.70 & 4459 & 3.0 & $6 \pm 12$ & -0.05 & 4339 & 2.4 & 6 & 0 & 26 & ${ }^{*} \mathrm{RC}$ \\
\hline $23 b$ & $15: 39: 16.369-50: 02: 49.33$ & 16.89 & 1.85 & 14.55 & 0.71 & 4594 & 66.9 & $9 \pm 18$ & -0.17 & 4019 & 1.9 & & & 23 & Mg-rich? \\
\hline $24 \mathrm{~b}$ & $15: 39: 03.749-50: 03: 49.83$ & 16.77 & 2.05 & 14.43 & 0.58 & 4451 & 82.8 & $-7 \pm 11$ & 0.15 & 4292 & 2.6 & 25 & 2 & 25 & ${ }^{*} \mathrm{RC}$ \\
\hline $25 b$ & $15: 39: 03.160-50: 02: 43.10$ & 17.34 & 1.44 & & & & 61.6 & $-3 \pm 18$ & 0.18 & 5576 & 4.2 & & & 28 & \\
\hline $26 \mathrm{~b}$ & 15:39:04.718-50:04:42.71 & 16.71 & 1.89 & 14.44 & 0.66 & 4652 & 23.8 & $-6 \pm 18$ & 0.15 & 4436 & 2.9 & & & 32 & ${ }^{*} \mathrm{RC}$ \\
\hline $27 \mathrm{~b}$ & $15: 39: 01.749-50: 03: 07.13$ & 15.64 & 2.17 & 12.99 & 0.79 & 4168 & 76.6 & $-2 \pm 13$ & 0.19 & 4126 & 1.9 & -1 & -9 & 44 & ${ }^{* *} \mathrm{RGB}, \mathrm{TiO}$ \\
\hline $28 \mathrm{~b}$ & $15: 39: 00.489-50: 03: 34.73$ & 15.99 & 2.02 & 13.43 & 0.73 & 4321 & 98.2 & $-2 \pm 7$ & 0.10 & 4178 & 2.5 & & & 39 & ${ }^{* *} \mathrm{RGB}$ \\
\hline $29 b$ & 15:39:01.008-50:04:11.73 & 17.11 & 1.79 & 14.85 & 0.62 & 4742 & 16.8 & $-10 \pm 15$ & 0.08 & 4770 & 2.3 & -2 . & -22 & 24 & ${ }^{*} \mathrm{RC}$ \\
\hline $30 \mathrm{~b}$ & $15: 39: 02.498-50: 02: 48.63$ & 16.97 & 1.82 & 14.19 & 1.03 & 4303 & 66.9 & $-1 \pm 17$ & 0.16 & 4573 & 2.5 & 26. & -5 & 26 & ${ }^{*} \mathrm{RC}$ \\
\hline $31 \mathrm{~b}$ & $15: 38: 54.032-50: 00: 17.88$ & 15.20 & 2.30 & 12.38 & 0.78 & 4003 & 11.5 & $-29 \pm 5$ & 0.17 & 4029 & 1.7 & & & 45 & ${ }^{* *} \mathrm{RGB}, \mathrm{TiO}$ \\
\hline $32 \mathrm{~b}$ & $15: 38: 58.105-50: 01: 33.20$ & 16.67 & 1.95 & 14.53 & 0.99 & 4712 & 32.5 & $-13 \pm 18$ & 0.04 & 4252 & 2.4 & 6 & -15 & 25 & ${ }^{*} \mathrm{RC}$ \\
\hline $33 \mathrm{~b}$ & $15: 38: 54.725-50: 01: 59.90$ & 16.93 & 1.82 & & & & 49.8 & $-90 \pm 15$ & -0.97 & 4420 & 1.8 & & & 29 & \\
\hline $34 \mathrm{~b}$ & $15: 38: 55.435-50: 04: 19.50$ & 16.43 & 1.97 & & & & 63.1 & $-49 \pm 11$ & 0.53 & 6992 & 0.0 & & & 37 & \\
\hline $35 \mathrm{~b}$ & $15: 38: 56.078-50: 02: 42.50$ & 16.95 & 1.83 & 13.94 & 0.68 & 4155 & 28.9 & $-6 \pm 17$ & 0.15 & 4581 & 2.4 & & & 24 & ${ }^{*} \mathrm{RC}$ \\
\hline $36 b$ & $15: 38: 57.308-50: 02: 59.60$ & 17.00 & 1.91 & 14.48 & 0.57 & 4422 & 17.6 & $-12 \pm 19$ & 0.18 & 4574 & 2.4 & & & 22 & ${ }^{*} \mathrm{RC}$ \\
\hline $37 \mathrm{~b}$ & $15: 38: 59.348-50: 03: 48.40$ & 16.79 & 1.89 & & & & 14.6 & $25 \pm 18$ & 0.49 & 6987 & 0.0 & 33 & -16 & 28 & \\
\hline $38 \mathrm{~b}$ & $15: 38: 56.585-50: 05: 06.38$ & 15.82 & 2.40 & 12.93 & 0.90 & 3933 & 86.1 & $27 \pm 13$ & 0.13 & 3945 & 2.1 & 5 & -11 & 41 & $\mathrm{RC} ?$ \\
\hline $39 b$ & $15: 38: 59.908-50: 04: 13.70$ & 15.24 & 2.14 & 14.50 & 1.40 & & 25.8 & $3 \pm 11$ & -0.03 & 4132 & 1.95 & 31 & -1 & 63 & ${ }^{* *} \mathrm{RGB}, \mathrm{TiO}$ \\
\hline
\end{tabular}


Table A.2. Lick indices ( $\lambda \leq 4531 \AA$; first line) measured in the summed spectrum of RC stars in BH176 and in the MILES medium-resolution spectrum of HD 184406 with uncertainties (second line indicated by the " \pm " sign) determined from bootstrapping of the object spectrum.

\begin{tabular}{|c|c|c|c|c|c|c|c|c|c|c|}
\hline ID & $\begin{array}{l}\mathrm{H} \delta_{\mathrm{A}} \\
(\AA)\end{array}$ & $\begin{array}{r}\mathrm{H} \gamma_{\mathrm{A}} \\
(\AA) \\
\end{array}$ & $\begin{array}{r}\mathrm{H} \delta_{\mathrm{F}} \\
(\AA) \\
(\AA)\end{array}$ & $\begin{array}{r}\mathrm{H} \gamma_{\mathrm{F}} \\
(\AA) \\
\end{array}$ & $\begin{array}{r}\mathrm{CN}_{1} \\
(\mathrm{mag}) \\
\end{array}$ & $\begin{array}{r}\mathrm{CN}_{2} \\
(\mathrm{mag}) \\
\end{array}$ & $\begin{array}{r}\mathrm{Ca} 4227 \\
(\AA) \\
\end{array}$ & $\begin{array}{r}\mathrm{G} 4300 \\
(\AA) \\
\end{array}$ & $\begin{array}{r}\mathrm{Fe} 4383 \\
(\AA) \\
\end{array}$ & $\begin{array}{r}\mathrm{Ca} 4455 \\
(\AA) \\
\end{array}$ \\
\hline $\mathrm{RC}$ in $\mathrm{BH} 176$ & -6.64 & -9.32 & -1.71 & -3.29 & 0.242 & 0.284 & 2.05 & 6.20 & 7.96 & 2.09 \\
\hline \pm & 0.35 & 0.32 & 0.20 & 0.22 & 0.008 & 0.01 & 0.22 & 0.39 & 0.30 & 0.17 \\
\hline \multirow[t]{2}{*}{ HD 184406} & -6.26 & -9.28 & -1.65 & -3.28 & 0.251 & 0.293 & 2.01 & 6.24 & 7.70 & 2.15 \\
\hline & 0.37 & 0.32 & 0.2 & 0.21 & 0.008 & 0.010 & 0.22 & 0.41 & 0.44 & 0.17 \\
\hline
\end{tabular}

Table A.3. Lick indices $(\lambda \geq 4531 \AA$ A; first line) measured in the summed spectrum of RC stars in BH176 and in the MILES medium-resolution spectrum of HD 184406 with uncertainties (second line indicated by the " \pm ” sign) determined from bootstrapping of the object spectrum.

\begin{tabular}{|c|c|c|c|c|c|c|c|c|c|c|}
\hline $\begin{array}{l}\text { ID } \\
(\mathrm{S} / \mathrm{N})\end{array}$ & $\begin{array}{l}\mathrm{Fe} 4531 \\
(\AA)\end{array}$ & $\begin{array}{r}\mathrm{Fe} 4668 \\
(\AA)\end{array}$ & $\begin{array}{l}\mathrm{H} \beta \\
(\AA)\end{array}$ & $\begin{array}{r}\mathrm{Fe} 5015 \\
(\AA)\end{array}$ & $\begin{array}{r}\mathrm{Mg}_{1} \\
(\mathrm{mag})\end{array}$ & $\begin{array}{r}\mathrm{Mg}_{2} \\
\text { (mag) }\end{array}$ & $\begin{array}{r}\operatorname{Mg} b \\
(\AA)\end{array}$ & $\begin{array}{r}\mathrm{Fe} 5270 \\
(\AA)\end{array}$ & $\begin{array}{r}\mathrm{Fe} 5335 \\
(\AA)\end{array}$ & $\begin{array}{r}\mathrm{Fe} 5406 \\
(\AA)\end{array}$ \\
\hline \multirow{2}{*}{$\mathrm{RC}$ in $\mathrm{BH} 176$} & 3.67 & 8.98 & 1.13 & 7.06 & 0.129 & 0.270 & 3.73 & 4.08 & 3.75 & 2.69 \\
\hline & 0.01 & 0.17 & 0.07 & 0.23 & 0.002 & 0.003 & 0.07 & 0.08 & 0.08 & 0.04 \\
\hline \multirow[t]{2}{*}{ HD 184406} & 3.70 & 8.71 & 1.12 & 7.12 & 0.146 & 0.283 & 3.57 & 4.12 & 3.73 & 2.69 \\
\hline & 0.11 & 0.17 & 0.03 & 0.23 & 0.002 & 0.003 & 0.07 & 0.08 & 0.09 & 0.04 \\
\hline
\end{tabular}

Table A.4. Open clusters older than 4 Gyr from the catalogue of Gozha et al. (2012). See Sect. 6 for details.

\begin{tabular}{lrrrrrrrr}
\hline \hline Cluster & $\begin{array}{r}M_{V_{0}} \\
(\mathrm{mag})\end{array}$ & $\begin{array}{r}E(B-V) \\
(\mathrm{mag})\end{array}$ & $\begin{array}{r}\text { Age } \\
(\mathrm{Gyr})\end{array}$ & $\begin{array}{r}{[\mathrm{Fe} / \mathrm{H}]} \\
(\mathrm{dex})\end{array}$ & $\begin{array}{r}{[\mathrm{Mg} / \mathrm{Fe}]} \\
(\mathrm{dex})\end{array}$ & $\begin{array}{r}Z \\
(\mathrm{kpc})\end{array}$ & $\begin{array}{r}\text { Dist }_{\mathrm{GC}} \\
(\mathrm{kpc})\end{array}$ & $\begin{array}{r}\text { Dist }_{\odot} \\
(\mathrm{kpc})\end{array}$ \\
\hline NGC 6791 & -4.95 & 0.14 & 4.4 & 0.32 & 0.13 & 1.11 & 8.17 & 5.85 \\
NGC 188 & -4.16 & 0.08 & 4.3 & 0.12 & 0.26 & 0.78 & 9.20 & 2.05 \\
King 2 & & 0.36 & 6.0 & -0.42 & & -0.47 & 12.12 & 5.75 \\
NGC 1193 & -1.08 & 0.21 & 5.0 & -0.22 & 0.23 & -0.96 & 12.03 & 4.57 \\
Berkeley 18 & & 0.54 & 4.3 & 0.02 & & 0.51 & 13.65 & 5.80 \\
Berkeley 70 & & 0.58 & 4.7 & -0.32 & & 0.26 & 12.09 & 4.17 \\
Berkeley 17 & & 0.68 & 10.0 & -0.10 & 0.12 & -0.17 & 10.69 & 2.70 \\
Trumpler 5 & -3.83 & 0.82 & 5.0 & -0.30 & & 0.04 & 10.25 & 2.40 \\
Berkeley 20 & & 0.22 & 6.0 & -0.61 & 0.22 & -2.51 & 15.88 & 8.40 \\
Collinder 106 & & 1.09 & 7.9 & & & -0.01 & 8.70 & 0.77 \\
Saurer 1 & & 0.15 & 5.0 & -0.38 & 0.05 & 1.70 & 20.26 & 13.20 \\
Berkeley 39 & & 0.12 & 7.9 & -0.21 & 0.15 & 0.84 & 11.90 & 4.78 \\
Berkeley 25 & & 0.40 & 5.0 & -0.20 & 0.11 & -1.92 & 17.82 & 11.40 \\
Collinder 261 & -2.61 & 0.40 & 8.9 & -0.03 & 0.14 & -0.21 & 7.10 & 2.19 \\
NGC 6253 & -1.85 & 0.32 & 5.0 & 0.43 & 0.01 & -0.17 & 6.61 & 1.58 \\
Berkeley 75 & & 0.10 & 4.0 & -0.22 & 0.38 & -1.77 & 15.17 & 9.10 \\
\hline
\end{tabular}


M. E. Sharina et al.: Gemini spectroscopy of the outer disk star cluster BH176
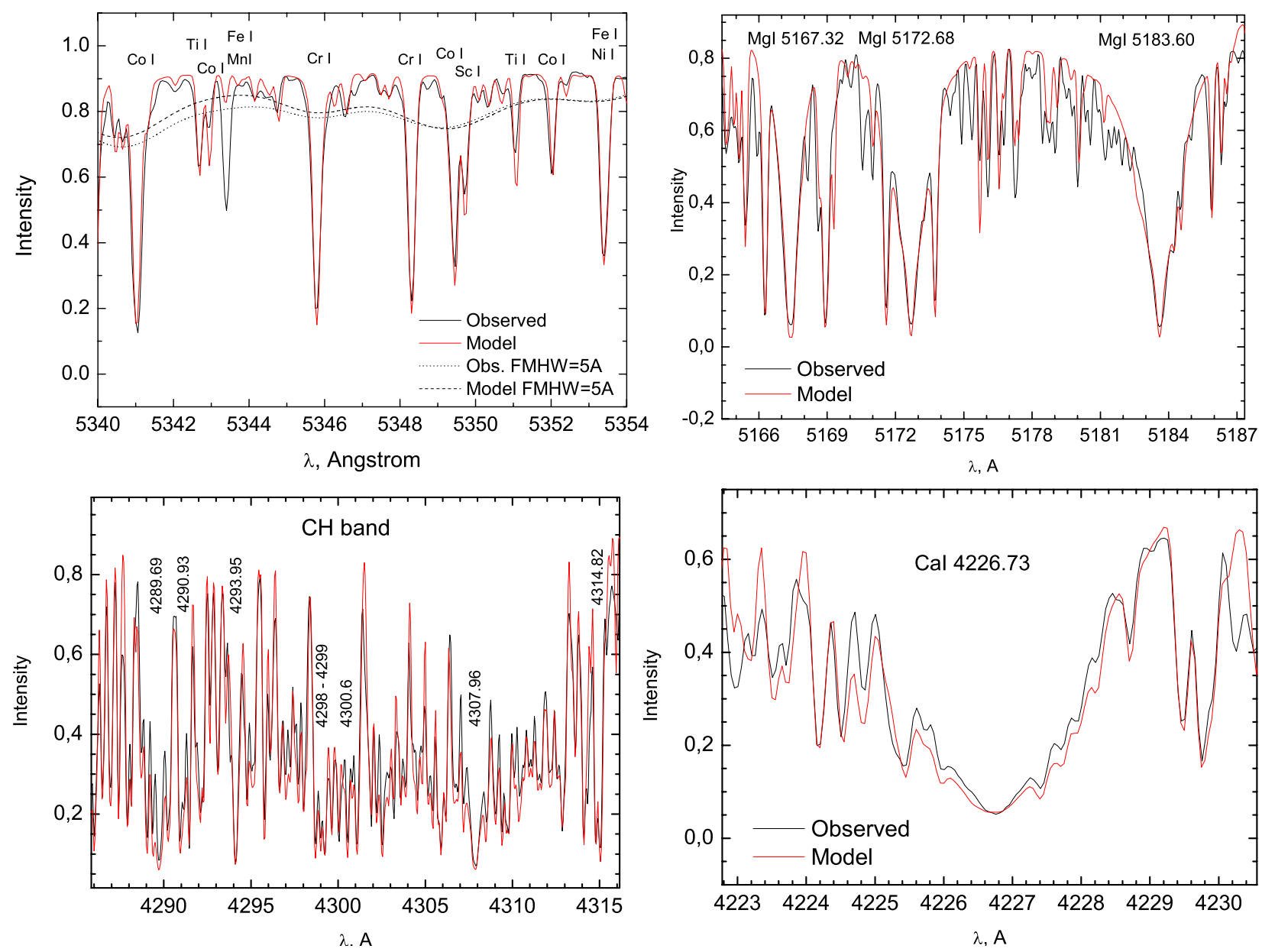

Fig. A.1. Comparison of the observed Arcturus spectrum from the ELODIE database and our calculated synthetic spectrum for a wavelength region including iron peak elements and for three wavelength regions corresponding to several prominent spectroscopic features $(\mathrm{CH}$ and $\mathrm{MgH}$ molecules, CaII H, K lines, CaI 4227 Å). The adopted parameters are from Ramírez \& Prieto (2011). 
A\&A 570, A48 (2014)
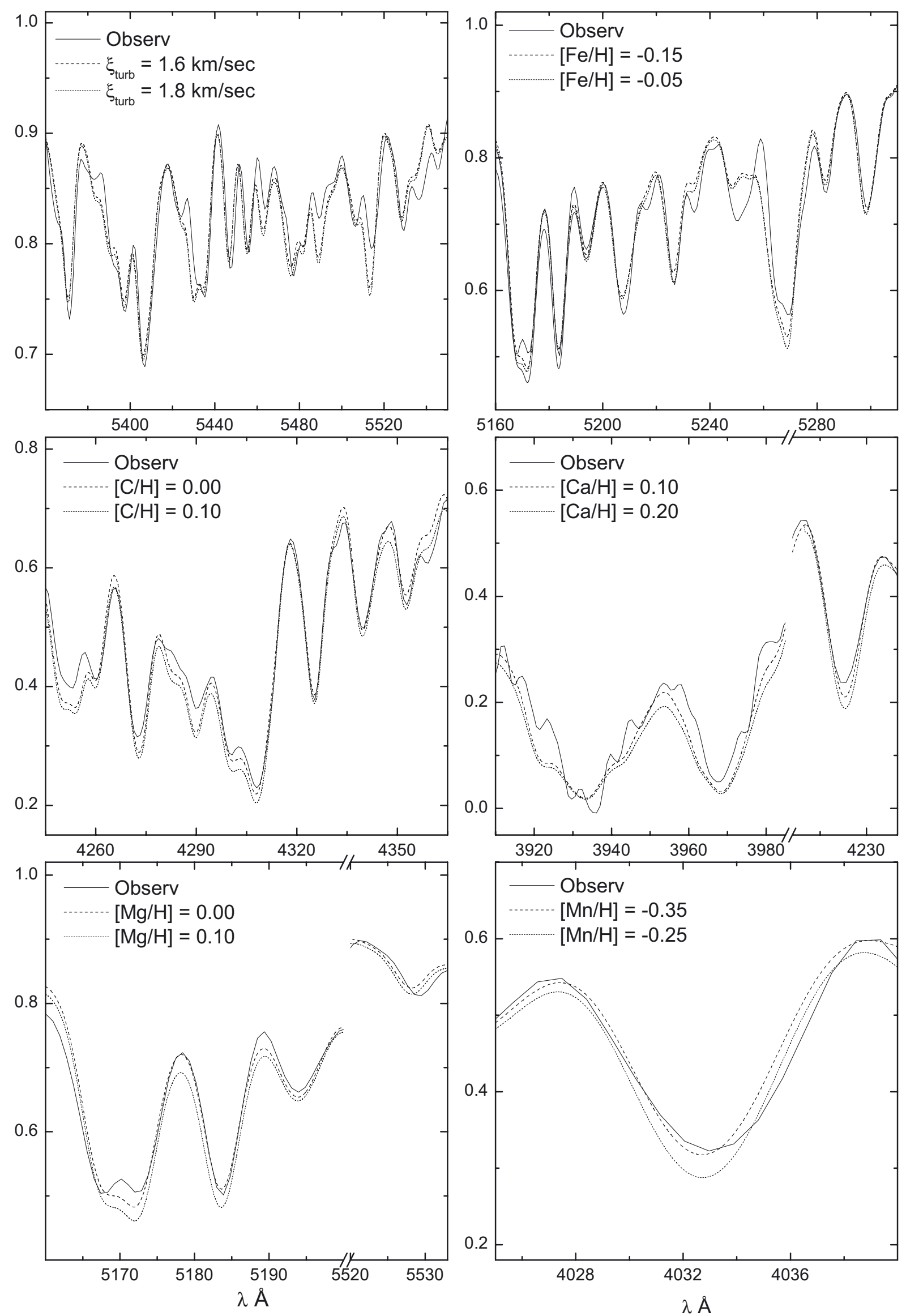

Fig. A.2. Illustration of fitting the abundances of different chemical elements (Sect. 3.2). The derived abundances are listed in Table 2. 
M. E. Sharina et al.: Gemini spectroscopy of the outer disk star cluster BH176
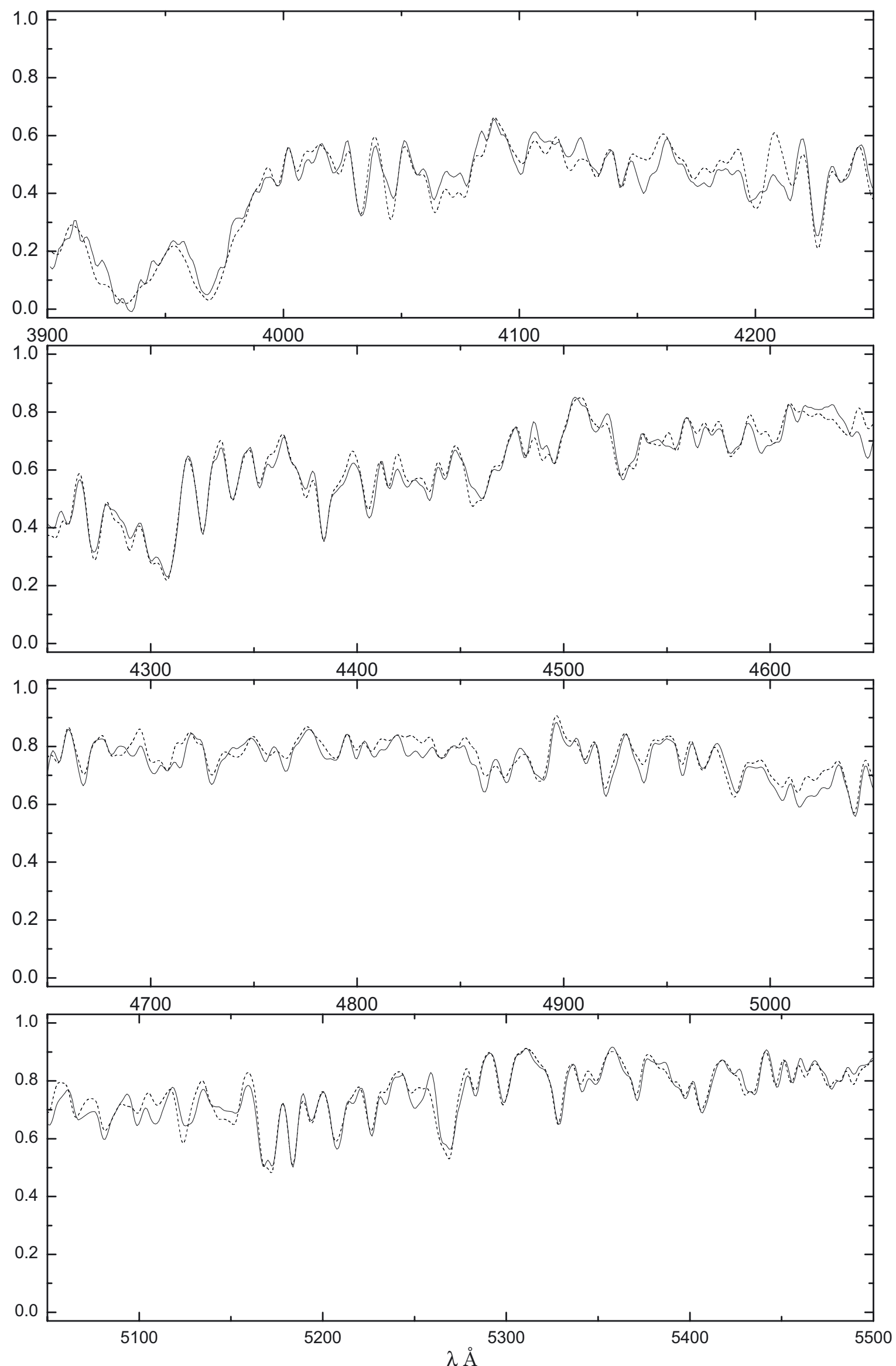

Fig. A.3. Fitting the whole spectrum of the RC stars in BH176 by the synthetic one (see Appendix A) The derived abundances are listed in Table 2. 